Tohoku J. exp. Med., 1969, 98, 33-63

\title{
Functional Model of Inner Medulla of Rabbit Kidney Based on Its Structural Principle*
}

\author{
Yasuhiko Sasaki and Norio Suwa \\ The First Department of Pathology (Prof. N. Suwa), \\ Tohoku University School of Medicine, Sendai
}

\begin{abstract}
The function of the inner medulla was analyzed on mathematical models with parameters of medullary structure. Three different theoretical assumptions were made on Henle's loops. The loops were regarded as an exchanger system of diffusion type, a counter-current multiplier system or a transport system with active sodium transport only on the ascending limb. The collecting tubule was treated as having active sodium transport and giving water to the interstitial tissue.

When Henle's loops were treated as a sodium-depriving system, the structure of the inner medulla characterized by quantitative predominance of Henle's loops over the collecting tubule toward the outer medulla acted synergetically to raise interstitial sodium concentration toward the medullary apex. In regard to the effect, the assumption of Henle's loops as a sodium-supplying system was conflicting with the medullary structure. The counter-current multiplier hypothesis of Henle's loops was not supported, because it presupposed the loops as a sodium-supplying system. Conversely, the model with Henle's loops as an exchanger system of diffusion type was found to be the most reasonable simulation of the inner medulla. A hypothesis of intramedullary sodium circulation involving both outer and inner medullas was proposed for the mechanism of water absorption from the collecting tubule or urinary concentration.
\end{abstract}

The role of the renal medulla in urinary concentration has been recognized from the observation that mammals with an ability of secreting urine of high osmolarity have large and long renal medullas. The first theoretical analysis of medullary function was the counter-current hypothesis proposed by Hargitay and Kuhn ${ }^{1}$ in 1951. In this hypothesis, the descending and ascending limbs of a Henle's loop are assumed to be separated by a semipermeable membrane. A part of water in glomerular filtrate flowing in the loop passes through the membrane from the descending to ascending limb on account of hydrostatic pressure difference. Sodium is left behind in the descending limb, so that progressive concentration of sodium toward the bend of the loop takes place. The high sodium concentration in the loop draws water from the interstitial tissue and further from the collecting tubule to cause urinary concentration.

According to this hypothesis urinary concentration in the renal medulla is a purely physical process without need for biological energy source in the medulla.

Received for publication, January 27, 1969.

* The Mechanism of Urinary Concentration in the Renal Medulla II. 
The energy necessary for urinary concentration is afforded by hydrostatic pressure, which has its origin in blood pressure generated by cardiac activity. However, as Hargitay and Kuhn pointed out, the pressure difference required to cause urinary concentration as experimentally observed would surpass the systemic blood pressure by several times. Consequently, it cannot be concluded that urinary concentration in the medulla is a simply physical process.

Taking this into consideration, Kuhn and Ramel ${ }^{2}$ stated in 1959 that the counter-current hypothesis would be sustained, if active transport of sodium was assumed from the ascending to descending limb instead of passive water transfer from the descending to ascending limb. The hypothesis appeared to find a general favor, because Wirz ${ }^{3}$ demonstrated experimentally remarkable elevation of osmolarity in Henle's loops and interstitial capillaries toward the medullary apex. Since then, high sodium concentration in the medullary interstitial tissue has been repeatedly ascertained by a number of investigators and has been interpreted in association with urinary concentration.

Although the counter-current hypothesis is apparently a plausible explanation of the mechanism of urinary concentration, the assumption of active sodium transport on Henle's thin segments does not seem to be sufficiently substantiated. The ascending and descending limbs appear morphologically indistinguishable from each other, and it is hardly to suppose that the one transfers sodium outward and the other inward by their capacity of active transport. A very important investigation in this respect was that of Marsh and Solomon ${ }^{4}$ in 1965. They stopped the flow of a Henle's thin segment with two separate oil droplets and examined the sodium concentration of fluid interposed between the droplets. Even in an observation for four hours they could not find a difference in sodium concentration between outside and inside the tubule. They concluded accordingly that Henle's thin segments had not a capacity of active sodium transport.

A quite different approach to the medullary function had been attempted by Hilger et $a l .{ }^{5}$ in 1959 . They examined the sodium concentration in the collecting tubules at different heights using a thin catheter, and found a progressively lowered sodium concentration toward the medullary apex. This was all the more noteworthy, because inulin concentration was found elevated toward the distal collecting tubule, indicating urinary concentration or water transfer to the interstitial tissue. They assumed therefore a strong active transport of sodium on the collecting tubule which must overwhelm the effect of water transfer. According to their result the collecting tubule would contribute much to sustaining high sodium concentration of the interstitial tissue. However, a theoretical model of urinary concentration based on this result has not yet been proposed.

The mechanism of urinary concentration has been hitherto investigated almost exclusively by physiologists, so that the structural principle of the total renal medulla does not seem to have drawn due attention. The theoretical treatment has been done on a simple model with a single loop and an unbranching collecting tubule, and the conclusion is directly correlated with experimental results. 
An approach in this way harbors a serious error, because the structural prineiple of the renal medulla is not taken into consideration at all. The inner medulla is composed of a pile of Henle's loops and a system of ramified collecting tubules, each of which has a regular structural principle, as revealed in a previous work of ours. ${ }^{6}$ The process in the actual renal medulla is an integration of the function of individual constituents according to the structural principle of the whole medulla. A theoretical assumption for example on a single Henle's loop which appears compatible with an experimental result may become utterly inappropriate or even absurd after integration. Conversely, a hypothesis on the function of individual constituents which seems contradictory to the observation may be sustained in the explanation of the total medullary function.

In the present study, theoretical models are proposed which have parameters of the structure of the whole inner medulla. On the function of individual constituents, simplest possible assumptions are made. They would not correspond to the real function exactly. However, so far as the intramedullary process can be reduced to the exchange of a certain substance, it is nevertheless possible to discuss the significance of the medullary structure on such a model. The purpose of the present study is not to reproduce the medullary function faithfully, but to elucidate the importance of the structure, which has been unduly neglected in renal physiology.

\section{Elementary Model}

Theoretical analysis is started from the simplest model of the inner medulla with a single Henle's loop of uniform size, an unbranching collecting tubule of uni-

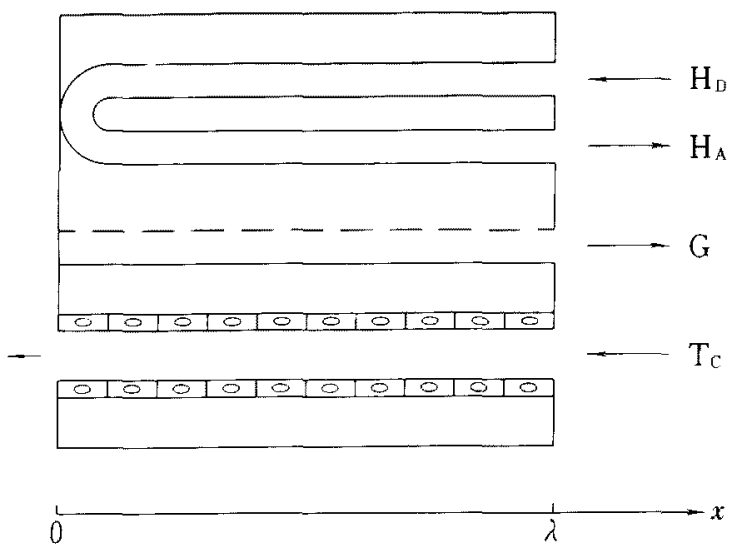

Fig. 1. An elementary model of the inner medulla is illustrated. It consists of a single Henle's loops with descending $\operatorname{limb} \mathrm{H}_{\mathrm{D}}$ and ascending $\operatorname{limb} \mathrm{H}_{\mathrm{A}}$, a capillary $\mathrm{G}_{\mathrm{H}}$ and a collecting tubule $\mathrm{T}_{\mathrm{C}}$. The arrows in the figure indicate the directions of flow in individual constituent structures. The tubules are supposed to lie parallel to a onedimensional scale and the bend of the loop is situated at the origin of the scale. The distance $x$ is taken from the bend of the loop toward the external border of the 'inner medulla'. The total length of the system is $\lambda$. For further explanations see text. 
form size and a capillary. The model is illustrated in Fig. 1. The comparison of such a simple model with a complex one, which will be considered later, is useful for making clear the influence of the structural principle of the inner medulla on the medullary function. The bend of the loop is supposed to be situated at the origin of a one-dimensional scale, and the loop and collecting tubule are layed parallel to the scale. A positive distance $x$ is taken from the origin to the outer medulla. At first, assumptions are made on the function of each constituent.

1) Henle's loop

The loop may have or may not have the capacity of active sodium transport, and both possibilities are taken into consideration. We assume that the loop transports actively a quantity $\gamma$ of sodium across a unit surface area of its wall per unit time. In this case, $\gamma$ is a constant independent of $x$. Absence of active transport can be expressed by taking $\gamma$ as zero. In the ascending limb active transport of sodium is directed outwards. In the descending limb the direction of transport must be reversed. We further assume that the loop is impermeable to water for the sake of easy mathematical treatment. In this case, the flow $V$ of the loop is a constant independent of $x$.

If active sodium transport is assumed on both limbs from the ascending to the descending, the loop constitutes a counter-current multiplier system. Writing sodium concentrations in the descending and ascending limbs $C_{D}$ and $C_{A}$ respectively, we obtain:

$$
\left\{\begin{array}{c}
\frac{d C_{D}}{d x}=-\frac{L_{h} \gamma}{V} \\
\frac{d C_{A}}{d x}=-\frac{L_{h} \gamma}{V}
\end{array}\right.
$$

where $L_{h}$ is the internal circumference of the loop. $C_{D}$ and $C_{A}$ are of course functions of $x$. Because $C_{D}$ and $C_{A}$ must be equal at the bend of the loop, we obtain upon integration:

$$
C_{D}=C_{A}=C_{D O}-\frac{L_{h} \gamma}{V} x=C_{A O}-\frac{L_{h} \gamma}{V} x
$$

in which $C_{D O}$ and $C_{A O}$ are the sodium concentration at the bend of the loop. If the total length of the loop is $\lambda$ and the sodium concentration at the proximal end of the descending limb is $\nu$, the above equation is transformed into:

$$
C_{D}=C_{A}=\nu+(\lambda-x) \frac{L_{h} \gamma}{V}
$$

Because $x$ is smaller than $\lambda$, a linear rise of intratubular sodium concentration toward the bend of the loop is demonstrated. 
So far, an interaction of intratubular and extratubular or interstitial sodium concentration has not been taken into consideration. Even if intratubular sodium concentration is elevated, it has no meaning whatever in raising the interstitial sodium concentration, when there is no passive sodium exchange between in and outside the tubule. We must therefore characterize Henle's loop as an exchanger system. In the present study, the loop is assumed to be an exchanger system of diffusion type: the quantity of sodium transferred across the wall of the loop per unit time is proportional to the difference of sodium concentrations between in and outside the tubule. If the sodium concentration of the interstitial tissue is $C$, the sodium exchange of this type can be expressed by the following differential equations:

$$
\left\{\begin{array}{l}
\frac{d C_{D}}{d x}=-\frac{L_{h} K_{s}}{V}\left(C-C_{D}\right) \\
\frac{d C_{A}}{d x}=\frac{L_{h} K_{s}}{V}\left(C-C_{A}\right)
\end{array}\right.
$$

where $K_{\mathrm{s}}$ is a permeability constant of the loop for sodium transfer. The derivation of the equation is explained in Fig. 2.

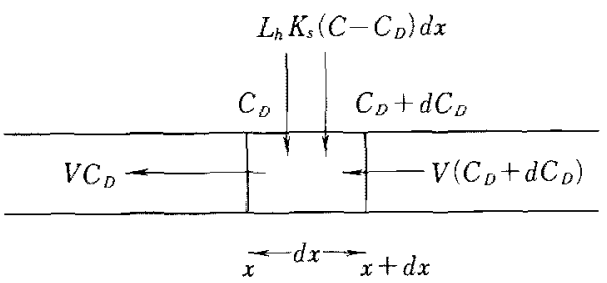

Fig. 2. The derivation of the differential equation on an exchanger system of diffusion type is explained. The figure represents a part of Henle's descending limb with a uniform perimeter $L_{h}$. The wall of the tubule is assumed to be impermeable to water, so that intratubular flow $V$ is regarded as a constant. Intratubular sodium concentrations at $x$ and $x+d x$ are supposed to be $C_{D}$ and $C_{D}+d C_{D}$ respectively. Extratubular sodium concentration is expressed by $C . K_{s}$ is the permeability constant for sodium. In a sufficiently small stretch between $x$ and $x+d x$ sodium quantity transferred into the tubule is:

$$
L_{h} K_{s}\left(C-C_{D}\right) d x .
$$

This quantity must be equal to the difference of sodium quantity carried into this stretch across $x+d x$ and that carried away across $x$. Because the direction of the flow is opposite to that of increasing $x$, the difference is written as:

$$
V C_{D}-V\left(C_{D}+d C_{D}\right) \text {. }
$$

Since (a) and (b) are equal, we obtain:

$$
V C_{D}-V\left(O_{D}+d C_{D}\right)=L_{h} K_{s}\left(C-C_{D}\right) d x
$$

and rearranging the expression:

$$
\frac{d C_{D}}{d x}=-\frac{L_{h} K_{S}}{V} \cdot\left(C-C_{D}\right) .
$$

The derivation on the ascending Iimb is essentially the same, the only difference being that the sign of the right side of the equation becomes positive. This is due to the same direction of intratubular flow and the increase of $x$. 
Now, we assume that the total balance of sodium between the loop and interstitial tissue is expressed by an algebraic sum of active and passive transport. In this case, the general equations for Henle's loop are:

$$
\left\{\begin{array}{l}
\frac{d C_{D}}{d x}=-\frac{L_{k} K_{s}}{V}\left(C-C_{D}\right)-\frac{L_{h} \gamma}{V} \\
\frac{d C_{A}}{d x}=\frac{L_{h} K_{s}}{V}\left(C-C_{A}\right)-\frac{L_{h} \gamma}{V} .
\end{array}\right.
$$

According to where active transport is presumed, some different combinations are obtained. If there is no active transport or if $\gamma$ is zero, the second term on the right side is omitted, and the equations for sodium exchange are simply (1) and (2). In this case, Henle's loop is merely an exchanger system of diffusion type without ability of active sodium transport.

When active transport is presumed on both limbs, the model corresponds to a counter-current multiplier system, and the equations are (3) and (4). Further it is possible to presume active sodium transport on only one of the limbs. Because sodium concentration is elevated toward the bend of the loop, it does not seem probable that descending limb provides sodium to the interstitial tissue. Consequently, the only possibility is the assumption of active sodium transport on the ascending limb. In this case, the ascending limb is called a transport system.

According to the above-mentioned three different assumptions on the function of Henle's loop, we obtain three different models. The difference, however, can be mathematically expressed as simple variations of a common form.

\section{2) Collecting tubule}

Two requirements must be met by the collecting tubule. Firstly, water must be transported from the tubule to the interstitial tissue or to interstitial capillaries. This corresponds to urinary concentration in the collecting tubule. The mechanism of water transport, however, is left undetermined at the start of mathematical derivation. We only assume that a quantity $W$ of water is transported from a unit internal surface area of the tubule per unit time. Generally speaking, $W$ is a function of $x$. However, in the elementary model now in question $W$ is treated as a constant, and later in the complex model an adequate function of $x$ is to substitute for $W$. Secondly, sodium is actively transported from the collecting tubule. This assumption is based on the experimental result of Hilger et al. Because water is simultaneously transported, we can express sodium transport with sodium concentration $T$ in fluid carried from the tubule to interstitial capillaries. $T$ is also a function of $x$, but it is treated preliminarily as a constant in the present simple model. In the complex model, which will be discussed later, it is replaced by an adequate function of $x$. The collecting tubule with such a function of sodium transport is also regarded as a transport system. 


\section{3) Capillaries}

In our model, Henle's loop is impermeable to water. Accordingly, water absorbed from the collecting tubule must be all carried away by the interstitial capillary, owing to high colloid osmotic pressure of the blood, if the diffusion of water in the direction of $x$ in the interstitial tissue is neglected. Because the capillary wall does not provide a barrier to sodium ion, we assume that water absorbed by the capillary contains sodium in a concentration $C$, which is exactly the same as that of the interstitial tissue. The capillary is thus regarded as an exchanger system of dialysis type.

With the characterization of the function of each constituent, three different combinations are possible. They are summarized as follows.
Model-1 $\left\{\begin{array}{l}\text { Henle's loop: Exchanger system of diffusion type } \\ \text { Collecting tubule: Sodium and water transport system } \\ \text { Capillary: Exchanger system of dialysis type }\end{array}\right.$
Model-2 $\left\{\begin{array}{c}\text { Henle's loop: Counter-current multiplier system and exchanger } \\ \text { system of diffusion type } \\ \text { Collecting tubule: Sodium and water transport system }\end{array}\right.$
Capillary: Exchanger system of dialysis type

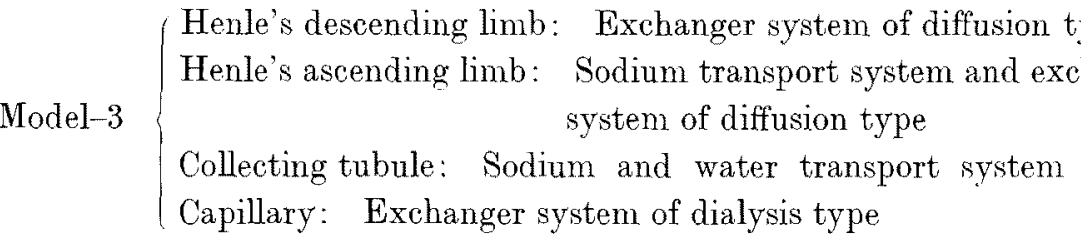

The difference of the above models is entirely due to different functional assumptions made on Henle's loop. The differential equations for Henle's loop in the three models are:

$$
\begin{aligned}
& \text { Model-1 }\left\{\begin{array}{l}
\frac{d C_{D}}{d x}=-\frac{L_{h} K_{s}}{\bar{V}} \cdot\left(C-C_{D}\right) \\
\frac{d C_{A}}{d x}=\frac{L_{h} K_{s}}{V}\left(C-C_{A}\right)
\end{array}\right. \\
& \text { Model-2 }\left\{\begin{array}{c}
\frac{d C_{D}}{d x}=-\frac{L_{h} K_{s}}{V} \cdot\left(C-C_{D}\right)-\frac{L_{h} \gamma}{V} \\
\frac{d C_{A}}{d x}=\frac{L_{h} K_{s}}{V}\left(C-C_{A}\right)-\frac{L_{h} \gamma}{V}
\end{array}\right. \\
& \text { Model-3 }\left\{\begin{array}{l}
\frac{d C_{D}}{d x}=-\frac{L_{h}}{V} K_{s} \\
V \\
\frac{d C_{A}}{d x}=\frac{L_{h} K_{s}}{V}\left(C-C_{D}\right)
\end{array}\left(C-C_{A}\right)-\frac{L_{h} \gamma}{V}\right.
\end{aligned}
$$


The differential equations are still insoluble in the form as they are. In order to make them soluble, another additional condition is required. We suppose now that the whole system is in a stationary state. Under this condition, the quantity of sodium carried away by the capillary out of the system must be equal to that given by Henle's loop and the collecting tubule to the interstitial tissue. The equation of equilibrium for a narrow zone between $x$ and $x+d x$ of the three models are:

Model-1: $\quad L_{c} W C \cdot d x=L_{h} K_{s}\left[\left(C_{D}-C\right)+\left(C_{A}-C\right)\right] d x+L_{c} W T \cdot d x$

Model-2: $\quad L_{c} W C \cdot d x=L_{b} K_{s}\left[\left(C_{D}-C\right)+\left(C_{A}-C\right)\right] d x+L_{c} W T \cdot d x$

Model-3: $\quad L_{c} W C \cdot d x=L_{h} K_{s}\left[\left(C_{D}-C\right)+\left(C_{A}-C\right)\right] d x+L_{h} \gamma \cdot d x$

$$
+L_{c} W T \cdot d x
$$

It is to be noticed that equations for Model-1 and Model-2 are the same, because in Model-2 the sodium quantity given from the ascending limb by active transport is totally taken by the descending limb, so that the quantity does not influence the equation of equilibrium. Rearranging the equations (11) to (13), we obtain:

$$
\begin{aligned}
& \text { Model-1: }\left(2+\frac{L_{c} W}{L_{k} K_{s}}\right) C-\left(C_{D}+C_{A}\right)=\frac{L_{c} W}{L_{k} K_{s}} \cdot T \\
& \text { Model-2: }\left(2+\frac{L_{c} W}{L_{h} K_{s}}\right) C-\left(C_{D}+C_{A}\right)=\frac{L_{c} W}{L_{h} K_{s}} \cdot T \\
& \text { Model-3: }\left(2+\frac{L_{c} W}{L_{h} K_{s}}\right) C-\left(C_{D}+C_{A}\right)=\frac{L_{c} W}{L_{h} K_{s}} \cdot T+\frac{\gamma}{K_{s}}
\end{aligned}
$$

When the differential equations from (14) to (16) are combined with the above equations of equilibrium of the corresponding models, a linear differential equation of the second order of $C$ is derived, which is common to all the three models.

$$
\begin{gathered}
\frac{d^{2} C}{d x^{2}}-\left(\begin{array}{c}
L_{h} K_{s} \\
V
\end{array}\right)^{2} \cdot \frac{L_{c} W}{2 L_{b} K_{s}+L_{c} W} \cdot C \\
=-\left(\frac{L_{h} K_{s}}{V}\right)^{2} \cdot \frac{L_{c} W}{2 L_{h} K_{s}+L_{c} W} \cdot T .
\end{gathered}
$$

The derivation is explained in the footnote.*)

*) Since mathematical treatments are the same in all the models, the derivation with Model-3 is described in this connection.

Upon differentiation of (16):

$$
\left(2+\frac{L_{c} W}{L_{h} K_{s}}\right) \frac{d C}{d x}-\left(\frac{d C_{D}}{d x}+\frac{d C_{A}}{d x}\right)=0
$$


Because the coefficients of (17) are constants, the equation is analytically soluble, and the general form of the solution is:

$$
C=M_{1} e^{b x}+M_{2} e^{-b x}+M_{3}
$$

in which $M_{1}, M_{2}$ and $M_{3}$ are constants and $b$ is given by:

$$
b=\frac{L_{h} K_{s}}{V} \cdot \frac{\sqrt{L_{c} W}}{\sqrt{2 L_{h} K_{s}+L_{c} W}} .
$$

The difference of the three models is revealed only in the difference of the coefficients $M_{1}$ and $M_{2}, M_{3}$ being zero. By substitution of

$$
\begin{aligned}
& p=\sqrt{2 L_{h} K_{s}+L_{c} W} \\
& q=\sqrt{L_{c} W}
\end{aligned}
$$

and writing $C$ at $x=0$ as $C_{0}$, we obtain the following solutions:

$$
\begin{aligned}
& \text { Model-1: } \quad C=T-\frac{1}{2}\left(T-C_{0}\right)\left(e^{b x}+e^{-b x}\right) \\
& \text { Model-2: } \quad C=T-\frac{1}{2}\left(T-C_{0}\right)\left(e^{b x}+e^{-b x}\right)-\frac{L_{k} \gamma}{p q}\left(e^{b x}-e^{-b x}\right) \\
& \text { Model-3: } \quad C=T-\frac{1}{2}\left(T-C_{0}\right)\left(e^{b x}+e^{-b x}\right)-\frac{L_{h} \gamma}{2 p q}\left(e^{b x}-e^{-b x}\right)
\end{aligned}
$$

Now, when $C$ in (5) to (10) are replaced by the solution of (20) to (22) according to each model, linear differential equations of the first order of $C_{A}$ and $C_{D}$ are obtained. They are again analytically soluble and the solutions are:

Substituting with (9) and (10):

$$
\left(2+\frac{L_{c} W}{L_{h} K_{s}}\right) \frac{d C}{d x}-\frac{L_{h} K_{s}}{V}\left(C_{D}-C_{A}\right)+\frac{L_{h} \gamma}{V}=0
$$

Upon differentiation again:

$$
\left(2+\frac{L_{c} W}{L_{h} K_{\mathrm{s}}}\right) \frac{d^{2} C}{d x^{2}}-\frac{L_{h} K_{\mathrm{s}}}{V}\left(\frac{d C_{D}}{d x}-\frac{d C_{A}}{d x}\right)=0
$$

Using (9) and (10):

$$
\left(2+\frac{L_{c} W}{L_{h} K_{s}}\right) \cdot \frac{d^{2} C}{d x^{2}}-\left(\frac{L_{h} K_{s}}{V}\right)^{2}\left[\left(C_{D}+C_{A}\right)-2 C\right]-\left(\begin{array}{c}
L_{h} K_{s} \\
V
\end{array}\right)^{2} \cdot \frac{\gamma}{K_{s}}=0
$$

By substitution with (16):

$$
\left(2+\frac{L_{c} W}{L_{h} \overline{K_{s}}}\right) \frac{d^{2} C}{d \overline{x^{2}}}-\left(\frac{L_{h} K_{s}}{V}\right)^{2}\left[\begin{array}{ll}
L_{c} W \\
L_{h} K_{s}
\end{array}(C-T) \cdot \frac{\gamma}{K_{s}}\right] \quad\left(\begin{array}{c}
L_{h} K_{s} \\
V
\end{array}\right)^{2} \cdot \frac{\gamma}{K_{s}}=0
$$

Rearranging the expression, we obtain:

$$
\frac{d^{2} C}{d x^{2}}-\left(\frac{L_{h} K_{s}}{V}\right)^{2} \cdot \frac{L_{c} W}{2 L_{h}} \frac{K_{s}+L_{c} W}{C} \cdot C=-\left(\frac{L_{h} K_{s}}{V}\right)^{2} \cdot \frac{L_{c} W}{2 L_{h} K_{s}+L_{c} W} \cdot T
$$


Model-1

$$
C_{D}=T-\frac{p}{2}\left(T-C_{0}\right)\left[\frac{e^{b x}}{p-q}+\frac{e^{-b x}}{p+q}\right]
$$

Model-2

$$
\begin{array}{r}
C_{A}=T-\frac{p}{2}\left(T-C_{0}\right)\left[\frac{e^{b x}}{p+q}+\frac{e^{-b x}}{p-q}\right] \\
C_{D}=T-\frac{p}{2}\left(T-C_{0}\right)\left[\frac{e^{b x}}{p-q}+\frac{e^{-b x}}{p+q}\right] \\
\quad-\frac{L_{h} \gamma}{q}\left[\frac{e^{b x}}{p-q}-\frac{e^{-b x}}{p+q}\right]+\frac{\gamma}{K_{\varsigma}}
\end{array}
$$

Model-3

$$
\begin{aligned}
C_{D}=T & -\frac{p}{2}\left(T-C_{0}\right)\left[\frac{e^{b x}}{p-q}+\frac{e^{-b x}}{p+q}\right] \\
& -\frac{L_{h} \gamma}{2 q}\left[\frac{e^{b_{x}}}{p-q}-\frac{e^{-b_{x}}}{p+q}\right]
\end{aligned}
$$$$
C_{A}=T-{ }_{2}^{p}\left(T-C_{0}\right)\left[\frac{e^{b x}}{p+q}+\frac{e^{-b x}}{p-q}\right]
$$$$
-\frac{L_{k} \gamma}{2 q}\left[\frac{e^{b x}}{p+q}-\frac{e^{-b x}}{p-q}\right]-\frac{\gamma}{K_{s}}
$$

These are the simplest forms of expression. However, $C_{\mathbf{0}}$ does not in reality represent an adequate initial condition of the differential equations. In its physical meaning $C_{0}$ is merely a result of equilibrium. The true initial condition must be sought in sodium concentration $\nu$ at the proximal end of Henle's descending limb. If the total length of the system is $\lambda$, we can rewrite the above results and the final expressions are:

Model-1 :

$$
\begin{aligned}
& C=T-(T-\nu) \cdot \frac{2 L_{h} K_{s}\left(e^{b x}+e^{-b x}\right)}{p\left[(p+q) e^{b \lambda}+(p-q) e^{-b \lambda}\right]}- \\
& C_{D}=T-(T-\nu) \cdot \begin{array}{l}
(p+q) e^{b x}+(p-q) e^{-b x} \\
(p+q) e^{b \lambda}+(p-q) e^{-b \lambda}
\end{array} \\
& C_{A}=T-(T-\nu) \cdot \frac{(p-q) e^{b x}+(p+q) e^{-b x}}{(p+q) e^{b \lambda}+(p-q) e^{-b \lambda}}
\end{aligned}
$$


Model-2:

$$
\begin{aligned}
& \begin{aligned}
C=T-\left[(T-\nu)+\frac{\gamma}{\overline{K_{s}}}\right] & \cdot \frac{2 L_{h} K_{s} \cdot\left(e^{b x}+e^{-b x}\right)}{p\left[(p+q) e^{b \lambda}+(p-q) e^{-b \lambda}\right]} \\
& +\frac{2 L_{k} \gamma \cdot\left[(p+q) e^{b(\lambda-x)}-(p-q) e^{-b(\lambda-x)}\right]}{p q\left[(p+q) e^{b \lambda}+(p-q) e^{-b \lambda}\right]}-\frac{\gamma}{p}
\end{aligned} \\
& C_{D}=T-\left[(T-\nu)+\frac{\gamma}{K_{s}}\right] \cdot \frac{(p+q) e^{b x}+(p-q) e^{-b x}}{(p+q) e^{b \lambda}+(p-q) e^{-b \lambda}} \\
& +\frac{2 L_{h} \gamma \cdot\left(e^{b(\lambda-x)}-e^{-b(\lambda-x)}\right)}{q\left[(p+q) e^{b \lambda}+(p-q) e^{-b \lambda}\right]}+\frac{\gamma}{K_{s}} \\
& C_{A}=T-\left[(T-\nu)+\frac{\gamma^{\prime}}{K_{s}}\right] \cdot \frac{(p-q) e^{b x}+(p+q) e^{-b x}}{(p+q) e^{b \lambda}+(p-q) e^{-b \lambda}} \\
& +\frac{\gamma \cdot\left[(p+q)^{2} e^{b(\lambda-x)}-(p-q)^{2} e^{-b(\lambda-x)}\right]}{K_{s} q\left[(p+q) e^{b \lambda}+(p-q) e^{-b \lambda}\right]}-\frac{\gamma}{K_{s}}
\end{aligned}
$$

Model-3:

$$
\begin{gathered}
C=T-(T-\nu) \cdot \frac{2 L_{h} K_{s}\left(e^{b x}+e^{-b x}\right)}{p\left[(p+q) e^{b \lambda}+(p-q) e^{-b \lambda}\right]} \\
\quad+\frac{L_{h} \gamma \cdot\left[(p+q) e^{b(\lambda-x)}-(p-q) e^{-b(\lambda-x)}\right]}{p q\left[(p+q) e^{b \lambda}+(p-q) e^{-b \lambda}\right]} \\
C_{D}=T-(T-\nu) \cdot \frac{(p+q) e^{b x}+(p-q) e^{-b x}}{(p+q) e^{b \lambda}+(p-q) e^{-b \lambda}} \\
+\frac{L_{h} \gamma \cdot\left(e^{b(\lambda-x)}-e^{-b(\lambda-x)}\right)}{q\left[(p+q) e^{b \lambda}+(p-q) e^{-b \lambda}\right]} \\
C_{A}=T-(T-\nu) \cdot \begin{array}{c}
(p-q) e^{b x}+(p+q) e^{-b x} \\
(p+q) e^{b \lambda}+(p-q) e^{-b \lambda}
\end{array} \\
\quad \gamma \cdot\left[(p+q)^{2} e^{b(\lambda-x)}-(p-q)^{2} e^{-b(\lambda-x)}\right]-\frac{\gamma}{K_{s}} \\
2 K_{s} q\left[(p+q) e^{b \lambda}+(p-q) e^{-b \lambda}\right]
\end{gathered}
$$

Before we draw the curves of the results, some analysis would be appropriate. In Model-1, $C$ and $C_{D}$ decrease steadily with increasing $x$, when a condition $T>\gamma$ is satisfied. The result is important because it demonstrates that a gradient of interstitial sodium concentration rising toward the medullary apex can be formed even in the absence of active sodium transport in Henle's loop, if the loop acts as an exchanger system of diffusion type. A counter-current multiplier system is not necessarily required in the formation of such a gradient. Experimental observation of interstitial sodium concentration rising toward the medullary apex does not lead to the conclusion that Henle's loops must have the ability of active sodium trans- 
port.

A second noteworthy result is that in Model-1 the sodium concentration in fluid absorbed from the collecting tubule must be higher than that at the proximal end of Henle's descending limb, in order that such a gradient of interstitial sodium concentration is formed. This is the most important restriction to Model-1 and its significance will be discussed later in the complex model.

A third characteristic of Model-1 is that $C_{A}$ is always higher than $C_{D}$. This means that in this model Henle's loop deprives the inner medulla of a certain sodium quantity when it leaves the inner medulla.

In Model-2 and Model-3 the conditions for $C$ to rise steadily toward the medullary apex are:

$$
\begin{gathered}
\text { Model-2: } T+\frac{\gamma}{K_{\mathrm{s}}}\left(1+\frac{p-q}{q} \cdot e^{-b \lambda}\right)>\nu \\
\text { Model-3: } T+\frac{\gamma}{K_{\mathrm{s}}} \cdot \frac{p-q}{q} \cdot e^{-b_{\lambda}}>\nu
\end{gathered}
$$

In these two models, it is not always required that $T$ is higher than $\nu$. This is because a certain sodium quantity is supplied from Henle's loop to the interstitial tissue and sustain its high sodium concentration.

For further understanding of the equations of the three models, it is better to examine the curves fixing the parameters to appropriate values. In Fig. 3 , the curves are illustrated giving the parameters a set of values. In each model a certain parameter was so adjusted that the sodium concentration of the interstitial tissue expressed in terms of $\mathrm{NaCl}$ concentration attained approximately $0.020 \mathrm{~g} / \mathrm{ml}$ at $x=0$. In Model-1 $T$ was adjusted. In Model-2 and Model-3, $T$ was fixed to $0.005 \mathrm{~g} / \mathrm{ml}$ or to a level which did not sustain high sodium concentration of the interstitial tissue by itself, and $\gamma$ was adjusted to produce the predetermined effect. The values of the other parameters were common to all the three models.

In all the models a sodium concentration gradient rising toward the medullary apex could be produced, although the pattern of the curves were considerably different according to models. It is noteworthy that among the three models only Model-2 had a counter-current multiplier system, but the other two did not. Nevertheless, the general pattern of experimentally observed gradient of interstitial sodium concentration was reproduced. However, the result of Fig. 3 is not a proper simulation of sodium concentration gradient in the actual renal medulla, because the structural principle is not yet taken into consideration. Nor is it possible to discuss the adequacy or inadequacy of theoretical assumptions made on each model. The pattern of Fig. 3 will be subjected to remarkable change, when the structural principle is incorporated in the model. This is revealed by complex models in the following part of this report. 

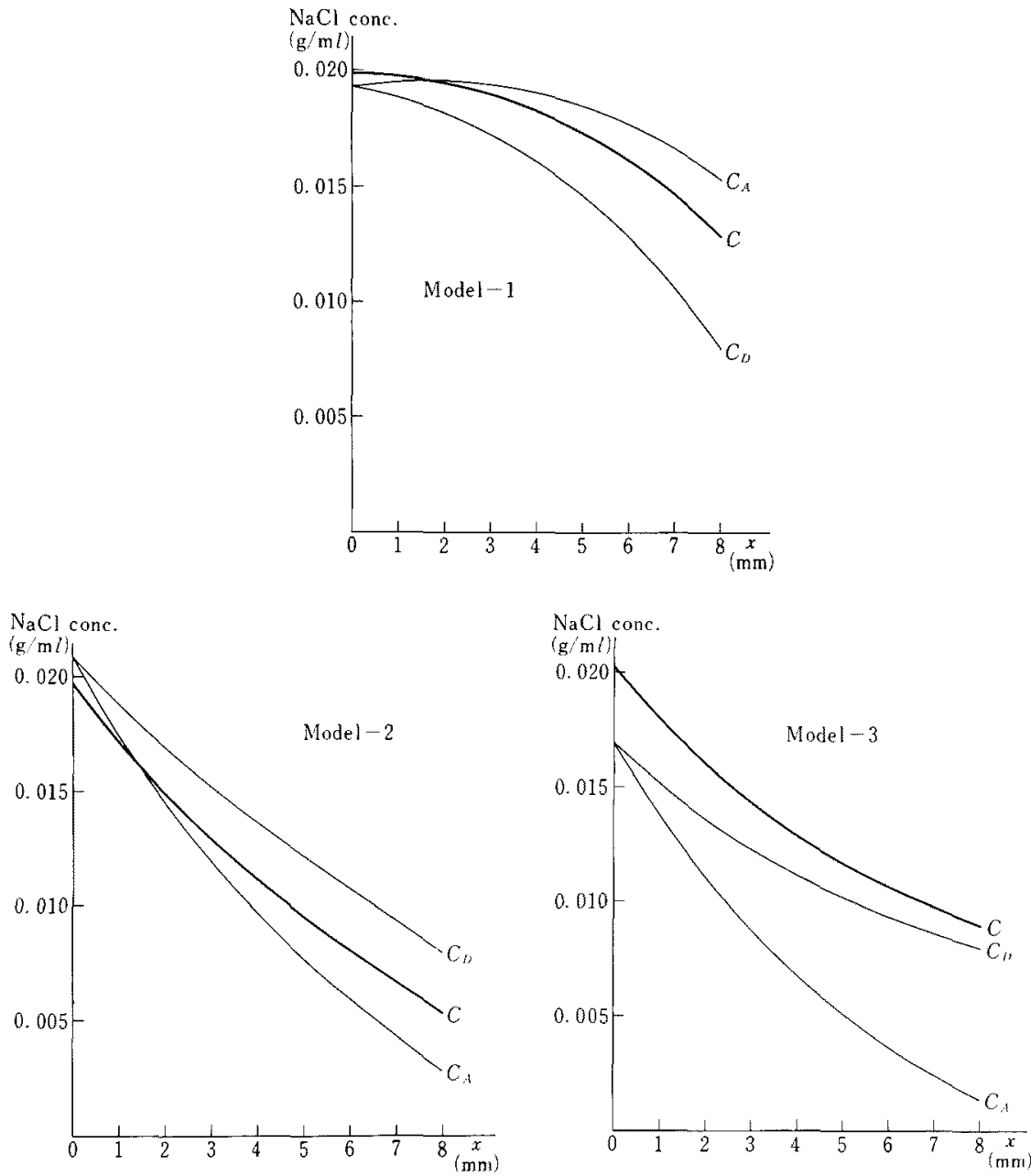

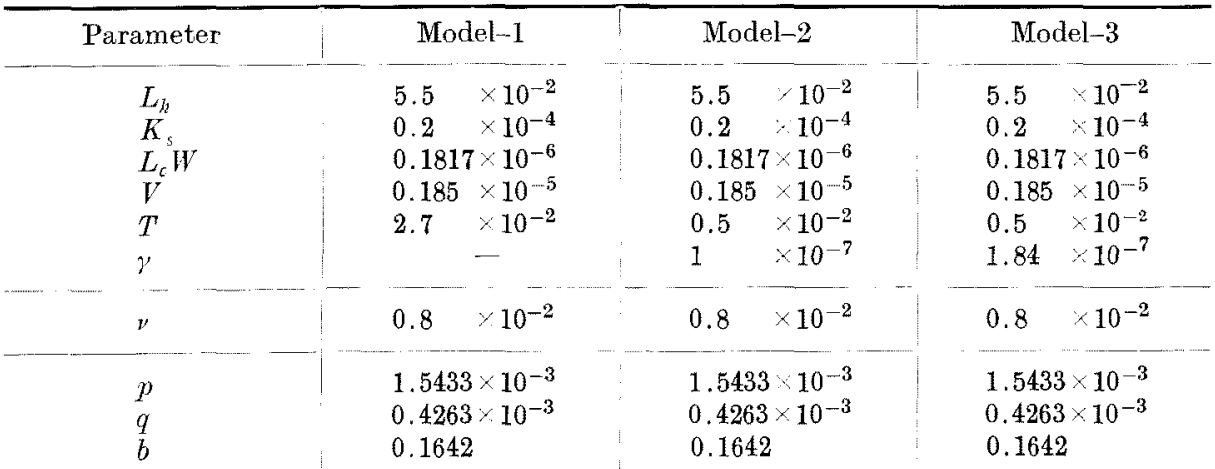

Fig. 3. An example of calculation on three elementary models is demonstrated. The parameter sets are given in the attached table. $T$ in Model-1 and $\gamma$ in Model-2 and Model-3 are so adjusted that $C_{0}$ or $C$ at $x=0$ attains approximately $0.020 \mathrm{~g} / \mathrm{ml}$ as $\mathrm{NaCl}$ concentration. For further explanations see text. 


\section{Complex Model with Parameters of Medullary Structure}

In a previous report of ours, attempts were made at quantitative estimation of the total internal circumferences of Henle's loops and collecting tubules at different heights of the inner medulla. The result could be expressed as:

and

$$
\begin{aligned}
& H(x)=H\left(e^{h x}-1\right) \\
& A(x)=A\left(e^{a x}-1\right)
\end{aligned}
$$

respectively. In the expressions, $x$ is a distance from the medullary apex to a certain point of the inner medulla along the medullary ray. The values of constants were determined from the measurements and the result was:

and

$$
\begin{aligned}
& H(x)=168\left(e^{0.60 x}-1\right) \\
& A(x)=114\left(e^{0.39 x}-1\right) .
\end{aligned}
$$

In the following part of the present report, the above result is incorporated in the model, and for this purpose mathematical expressions of the elementary model must be transformed.

\section{1) Henle's loop}

If we assume that sodium concentrations at the proximal end of the descending limbs are equal, the differential equations (5), (7) and (9) can be used without any correction in the complex model, and $C_{D}$ at the same $x$ is equal in all the descending limbs.

The differential equations of the ascending limbs, however, are different from those in the model of a single loop. In the pile of Henle's loops the descending limbs

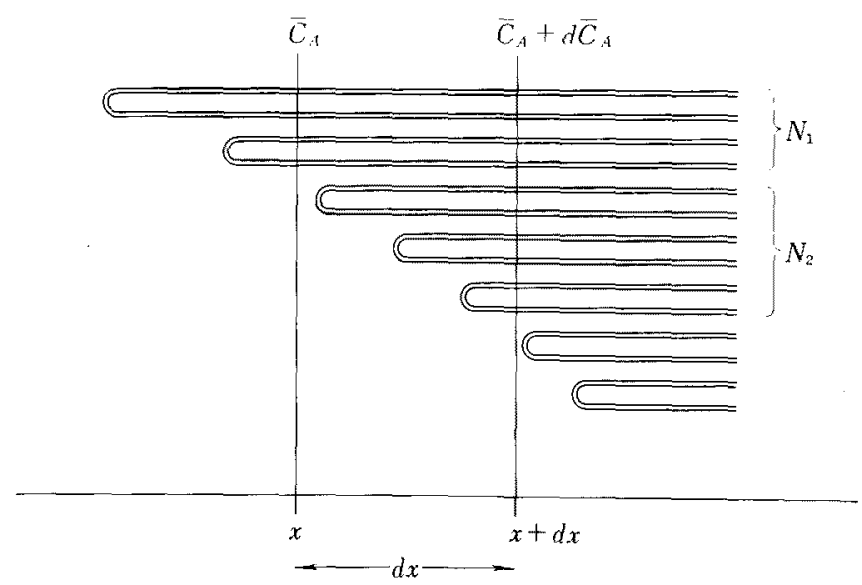

Fig. 4. The pile of Henle's loops is illustrated in reference to the derivation of the differential equation of the ascending limbs. $N_{1}$ refers to the loops which have made turn before $x$. In this group of loops intratubular sodium concentration changes as a result of sodium exchange. In $N_{2}$ the loops turn in the small stretch between $x$ and $x+d x$. They influence $\bar{C}_{A}$ or mean $C_{A}$ by entering the stretch with a sodium concentration of $C_{D}$. The influence of sodium exchange of the loops is negligible, because it becomes an infinitesimal of higher order, when $d x$ is infinitely small. 
turn to the ascending at different heights. Consequently, $C_{A}$ is not the same even at the same $x$. Because passive sodium exchange of ascending limbs with the interstitial tissue depends upon the difference $\left(C-C_{A}\right)$, we can use $\vec{C}_{A}$, the arithmetical mean of $C_{A}$, in the equation for all the ascending limbs. In this case, $d \bar{C}_{A} / d x$ is not simply determined by passive sodium exchange and active sodium transport alone.

When we consider a very small range between $x$ and $x+d x, \bar{C}_{A}$ in this range is influenced by two different processes. In the ascending limbs of the loops which have already made turn before $x$, the change of $\bar{C}_{A}$ is due to passive and active sodium exchange, as in the case of a single loop, and the same differential equation is to be applied. When the increase in $\bar{C}_{A}$ of this origin is expressed by $d \bar{C}_{A A}$, we can write:

$$
\begin{gathered}
\text { Model-1: } \frac{d \bar{C}_{A A}}{d x}=\frac{L_{h} K_{s}}{V}\left(C-\bar{C}_{A}\right) \\
\left\{\begin{array}{l}
\text { Model-2: } \quad \frac{d \bar{C}_{A A}}{d x}=\frac{L_{h} K_{s}}{V}\left(C-\bar{C}_{A}\right)-\frac{L_{h} \gamma^{\prime}}{V} \\
\text { Model-3: }
\end{array}\right.
\end{gathered}
$$

On the other hand, $\bar{C}_{A}$ is also influenced in this range by the other different process. This is because a certain small number of Henle's loops turn in this range from the descending to ascending limbs. The number $N_{h}$ of the loop increases in the course of $d x$. As $N_{h}$ is equal to $H(x) / 2 L_{k}$, the increase in the number $d N_{k}$ of Henle's loops in the course of $d x$ from $x$ is given by:

$$
d N_{h}=\frac{1}{2 L_{h}} \cdot \frac{d H(x)}{d x} \cdot d x=\frac{H}{2 L_{h}} \cdot h e^{h x} \cdot d x
$$

These loops enter the range with a sodium concentration of $C_{D}$, and their influence on $\bar{C}_{A}$ is determined by the product of $d N_{F}$ and the difference $\left(C_{D}-\bar{C}_{A}\right)$. The increase in $\bar{C}_{A}, d \bar{C}_{A}$, in the course of $d x$ from $x$ is consequently given by:

$$
d \bar{C}_{A}=\left[N_{h} \cdot d \bar{C}_{A A}+\left(C_{D}-\bar{C}_{A}\right) \cdot d N_{h}\right] / N_{h}
$$

From this we obtain:

$$
\begin{gathered}
\text { Model-1: } \frac{d \bar{C}_{A}}{d x}=\frac{L_{h} K_{s}}{V}\left(C-\bar{C}_{A}\right)+h\left(1+\frac{1}{e^{h x}-1}\right)\left(C_{D}-\bar{C}_{A}\right) \\
\left\{\begin{array}{l}
\text { Model-2: } \\
\text { Model-3: }
\end{array} \frac{d \bar{C}_{A}}{d x}=\frac{L_{h} K_{s}}{V}\left(C-\bar{C}_{A}\right)+h\left(1+\frac{1}{e^{h x}-1}\right)\left(C_{D}-\bar{C}_{A}\right)-\frac{L_{h} \gamma}{V}\right.
\end{gathered}
$$

\section{2) Equation of sodium equilibrium}

There is no theoretical difficulty in the equation of sodium equilibrium at $x$. It is only necessary to multiply the terms of the equations on the simple model by 
$H(x) / 2$ or $A(x)$. In the complex model, however, it is no more appropriate to regard $W$ and $T$ as constants, and they are written as functions of $x$ in the form of $W(x)$ and $T(x)$ respectively. The equations are accordingly:

$$
\left\{\begin{aligned}
\text { Model-1: } & \frac{H}{2}\left(e^{h x}-1\right) \cdot K_{\mathrm{s}}\left(2 C-C_{D}-\bar{C}_{A}\right)+A\left(e^{a x}-1\right) \cdot W(x) C \\
\text { Model-2: } & =A\left(e^{a x}-1\right) \cdot W(x) T(x)
\end{aligned}\right.
$$

Model-3: $\quad \underset{2}{H}\left(e^{h x}-1\right) \cdot K_{s}\left(2 C-C_{D}-\bar{C}_{A}\right)+A\left(e^{a x}-1\right) \cdot W(x) C$

$$
=A\left(e^{a x}-1\right) W(x) T(x)+{ }_{2}^{H}\left(e^{i x}-1\right) \cdot \gamma
$$

At present we have no means to define the functions $W(x)$ and $T(x)$ by experimental data. Consequently, we must be satisfied in the present state to apply some convenient functions of $x$, in order to examine the effect of increasing or decreasing $W(x)$ or $T(x)$ with increasing $x$ on the result of calculation. In the following part of the present report $W(x)$ and $T(x)$ are represented by the following exponential functions:

$$
W(x)=W e^{\omega x}
$$

and

$$
T(x)=T e^{\tau x}
$$

in which $W, \omega, T$ and $\tau$ are constants. The estimation of these parameters will be discussed later.

It is impossible in the case of our complex model to derive differential equations of $C$ alone. The differential equations of Henle's loops and the equation of sodium equilibrium are transformed into the following expressions and submitted to computer analysis.

Model-1 :

$$
\begin{aligned}
& \frac{d C_{D}}{d x}=-\frac{L_{h} K_{s}}{V} \cdot\left(C-C_{D}\right) \\
& \frac{d C_{A}}{d x}=\frac{L_{h} K_{s}}{V}\left(C-\bar{C}_{A}\right)+h\left(1+\frac{1}{e^{h x}-1}\right)\left(C_{D}-\bar{C}_{A}\right) \\
& C=\quad \begin{array}{c}
\left(C_{D}+\bar{C}_{A}\right)+\frac{2 T W A}{K_{s} H} \cdot \frac{e^{(\omega+\tau) x} \cdot\left(e^{a x}-1\right)}{\left(e^{h x}-1\right)} \\
2\left(1+\frac{W A}{K_{s} H^{-}} \cdot \frac{e^{\omega_{x}}\left(e^{a x}-1\right)}{\left(e^{h x}-1\right)}\right)
\end{array} \\
& \text { At } x=0, \quad \frac{d \bar{C}_{A}}{d x}=0
\end{aligned}
$$


Model-2:

$$
\left\{\begin{array}{l}
\frac{d C_{D}}{d x}=-\frac{L_{h} K_{s}}{V}\left(C-C_{D}\right)-\frac{L_{h} \gamma}{V} \\
\frac{d \bar{C}_{A}}{d x}=\frac{L_{h} K_{s}}{V}\left(C-\bar{C}_{A}\right)+h\left(1+\frac{1}{e^{h x}-1}\right)\left(C_{D}-\bar{C}_{A}\right)-\frac{L_{h} \gamma}{V} \\
C=\frac{\left(C_{D}+\bar{C}_{A}\right)+\frac{2 T W A}{K_{s} H} \cdot \frac{e^{(\omega+\tau) x} \cdot\left(e^{a_{x}}-1\right)}{\left(e^{h x}-1\right)}}{2\left(1+\frac{W A}{K_{s} H} \cdot \frac{e^{\omega x} \cdot\left(e^{a_{x}}-1\right)}{\left(e^{h x}-1\right)}\right)} \\
\text { At } x=0, \quad \frac{d \bar{C}_{A}}{d x}=-\frac{L_{h} \gamma}{V}
\end{array}\right.
$$

Model-3:

$$
\left\{\begin{array}{c}
\frac{d C_{D}}{d x}=-\frac{L_{h} K_{\mathrm{s}}}{V}\left(C-C_{D}\right) \\
\frac{d \bar{C}_{A}}{d x}=\frac{L_{h} K_{s}}{V}\left(C-\bar{C}_{A}\right)+h\left(1+\frac{1}{e^{h x}-1}\right)\left(C_{D}-\bar{C}_{A}\right)-\frac{L_{h} \gamma}{V} \\
C=\frac{\left(C_{D}+\bar{C}_{A}\right)+\frac{\gamma}{K_{\mathrm{s}}}+\frac{2 T W A}{K_{\mathrm{s}} H} \cdot \frac{e^{(\omega+\gamma) x} \cdot\left(e^{a_{x}}-1\right)}{\left(e^{h x}-1\right)}}{\left.21+\frac{W A}{K_{\mathrm{s}} H} \cdot \frac{e^{\omega x}\left(e^{a x}-1\right)}{\left(e^{h x}-1\right)}\right)} \\
\text { At } x=0, \quad \frac{d \bar{C}_{A}}{d x}=-\frac{L_{h} \gamma}{V}
\end{array}\right.
$$

From a theoretical viewpoint it would be desirable to derive a differential equation, in which sodium concentration $\nu$ at the proximal end of the descending limb can be used as the initial condition. However, such a derivation is apparently impossible, and we are forced to use $C_{0}$, sodium concentration of the interstitial tissue at $x=0$ as the initial condition. With each set of parameters, a series of values are given to $C_{0}$ and the results of computation is assessed by the value of obtained $\nu$ : from the results only those are adopted, in which $\nu$ is sufficiently close to a predetermined value. In some situation, another way of computation may be more convenient. In this case, $C_{0}$ is fixed to a predetermined value and a series of values are given to one of the parameters, the other parameters being fixed to a given set of values. In this method too, only those results are taken, in which $\nu$ is sufficiently close to a predetermined value. In the present study, $T$ is let change in Model-1, and $\gamma$ in Model-2 and Model-3. Before the results of computation are presented, some comments will be necessary for the determination of the parameters. 


\section{Determination of Parameters}

Our models have 12 parameters, $H, h, A, a, L_{k}, V, W, \omega, T, \tau, \gamma$ and $K_{s}$. The first five are structural parameters and were already determined in a previous report of ours. The other seven are physiological or physical ones. They are either subjected to some physiological variations or utterly unknown. For the latter group of parameters some consideration seems necessary.

$V$ : The flow of each Henle's loop was estimated at $V=1.85 \times 10^{-6} \mathrm{ml} / \mathrm{min}$ for the standard condition. The value is obtained as follows. Glomerular filtration rate of the rabbit is about $6 \mathrm{ml} / \mathrm{min}$, and on a kidney it is $3 \mathrm{ml} / \mathrm{min}$, of which $1 / 5$ is regarded to How into the Henle's loops. On the other hand, the total number of nephrons in a rabbit's kidney was about 270,000 to 300,000 according to our own investigation. In the present study the value of 270,000 was used, and $V$ was calculated as follows.

$$
V=3 \times(1 / 6) \times(1 / 2.7) \times 10^{-5} \mathrm{ml} / \mathrm{min}=1.85 \times 10^{-6} \mathrm{ml} / \mathrm{min}
$$

$V$ may be subjected to physiological variation and it is possible to examine its effect on the medullary function with the model.

$W$ and $\omega$ : There is to-day no reliable experimental result available on $W$ and $\omega$. However, we can roughly estimate a reasonable range. Urinary volume of the rabbit is generally regarded as $0.15-0.3 \mathrm{ml} / \mathrm{min}$ per kidney. The values correspond to 'average' urinary volume, and in a state of urinary concentration it would be appropriate to presume a much lower urinary volume of $0.05-0.1 \mathrm{ml} / \mathrm{min}$ per kidney. Now it is generally accepted that about $5 \%$ of GFR or about $0.15 \mathrm{ml} / \mathrm{min}$ flow into the medulla. How much water is absorbed in the inner medulla out of this quantity is unknown. However, we assume in the present study that $0.05 \mathrm{ml} / \mathrm{min}$ is absorbed in the outer medulla, another $0.05 \mathrm{ml} / \mathrm{min}$ in the inner medulla and the third $0.05 \mathrm{ml} / \mathrm{min}$ is excreted as final urine. When $\Delta Q$ is the quantity of water absorbed in the inner medulla, it is determined by the following relation:

$$
\Delta Q=\int_{0}^{8} W e^{a x} \cdot A\left(e^{a x}-1\right) d x
$$

the total length of the inner medulla being $8 \mathrm{~mm}$. When $\Delta Q$ is taken as $0.05 \mathrm{ml} / \mathrm{min}$ and one of $W$ and $w$ is given, the other is determined from (18). In the present study, the following two combinations are used.

$$
\begin{aligned}
& \omega=0, \text { and } W=9.238 \times 10^{-6} \mathrm{ml} / \mathrm{min} \\
& \omega=-0.1, \text { and } W=1.678 \times 10^{-5} \mathrm{ml} / \mathrm{min} .
\end{aligned}
$$

In the former, $W(x)$ is a constant. This combination is most appropriate in revealing the significance of the medullary structure. The latter combination takes into consideration that water absorption from the collecting tubule may be in some way or other associated with the difference of sodium concentration in and outside the tubule. Because the difference is expected to be larger toward the medullary apex, $W(x)$ is made to decrease with increasing $x$.

$T$ and $\tau$ : In Model-1, a restriction is given to $T$ and $\tau$ by expected sodium concentration of the interstitial tissue at the medullary apex. $T$ cannot be lower than that, as was already demonstrated. Under this condition there is also an inevitable restriction to $\tau$. When the total quantity of sodium transported from the collecting tubule of the inner medulla to the interstitial tissue is $\Delta N a$, there is the following relation:

$$
\Delta N a=\int_{0}^{8} W T e^{(r+w) x} \cdot A\left(e^{a x}-1\right) d x
$$

It is evident that $\Delta N a$ increases with increasing $\tau$. This sodium quantity must be again afforded by glomerular filtrate flowing into the inner medulla. To increase $\triangle N a$, the urine at the external border of the inner medulla has to become increasingly hypertonic. Under the condition that the final urinary volume is $0.05 \mathrm{ml} / \mathrm{min}$ per kidney and $T$ is 0.020 $\mathrm{g} / \mathrm{ml}$ in terms of $\mathrm{NaCl}$ concentration, it is calculated that $\mathrm{NaCl}$ concentration in the collect- 
ing tubule at the external border of the inner medulla must be higher than $0.018 \mathrm{~g} / \mathrm{m} \mathrm{l}$, when

$\tau$ attains 0.1. It seems hardly necessary to use a higher value of $\tau$.

In Model-2 and Model-3 high sodium concentration of the interstitial tissue depends to the major part on the sodium quantity afforded by Henle's loops. It is not required that the collecting tubule transports sodium in such a high concentration as in Model-1. The restriction to $T$ and $\tau$ is given in Model-2 and Model-3 by that $T(x)$ cannot be lower than sodium concentration $C_{c}$ in the collecting tubule. Otherwise, $C_{c}$ would rise toward the medullary apex, which is conflicting with experimental observation. Practically it would be appropriate to set the lower limit of $T(x)$ at $0.005 \mathrm{~g} / \mathrm{ml}$ in terms of $\mathrm{NaCl}$ concentration.

$\gamma$ : There is of course no direct measurement available on $\gamma$, the quantity of active sodium transport from unit surface area of Henle's loops per unit time. It is only possible in our present theoretical analysis to adjust $\gamma$, so that a predetermined interstitial sodiumconcentration is attained in the result of computation.

$K s$ : No reliable experimental data are to-day available on this parameter, the permeability of Henle's loop to sodium. It can be only so adjusted that the results are 'reasonable'. In the present models, the possible range of $K s$ is taken as $2-4 \times 10^{-5} \mathrm{~g} / \mathrm{mm}^{2} / \mathrm{min}$ in terms of $\mathrm{NaCl}$ in the presence of unit cencentration difference of $1 \mathrm{~g} / \mathrm{ml}$ of $\mathrm{NaCl}$ between in and outside the loop.

\section{Results of Computer Analysis}

A number of representative patterns obtained by computation are presented in Fig. 5. The set of parameters is given in tables attached to figures. Throughout the presented cases $A, a, H, h$ and $V$, that is, structural parameters and the flow of Henle's loop, are fixed to a common set. In all the cases $C_{0}$ and $\nu$ are the same and set to $0.020 \mathrm{~g} / \mathrm{ml}$ and $0.008 \mathrm{~g} / \mathrm{ml}$ of $\mathrm{NaCl}$ respectively. Consequently, one of the parameters loses freedom. In Model-1 the parameter is $T$, and in Model-2 and Model-3, $\nu$.

Three combinations of $\omega$ and $\tau$ are considered.

$\omega=0$ and $\tau=0 ;$ In this combination $W(x)$ and $T(x)$ are constants. This condition is most adequate in revealing the influence of medullary structure.

$\omega=-0.1$ and $\tau=0$ : In this combination water absorption from the collecting tubule becomes smaller toward the external border of the inner medulla. $T(x)$ is a constant. Consequently, sodium transport is also lowered toward the external border of the inner medulla.

$\omega=-0.1$ and $\tau=0.1$ : This combination presumes constant active sodium transport of the collecting tubule on its entire length, but lowered water absorption toward the the external border of the inner medulla.

In the latter two combinations water absorption from the collecting tubule is regarded as correlated in some way with the difference in sodium concentrations between the interstitial tissue and intratubular urine.

In all the models some sets of parameters could be found which satisfied the requirement to elevate $\mathrm{NaCl}$ concentration of the interstitial tissue to $0.020 \mathrm{~g} / \mathrm{ml}$ at the medullary apex, while $\mathrm{NaCl}$ concentration at the proximal end of the descending limbs of Henle is fixed to $0.008 \mathrm{~g} / \mathrm{ml}$. Even in the complex models with parameters of medullary structure the pattern of experimentally observed gradient of sodium concentration could be reproduced, irrespective of the presence or absence of a counter-current multiplier system. Such an effect is brought about by the character of Henle's loops as an exchanger system of diffusion type. So far there is no definite reason to select one of the models as the most reasonable one. 
We can only suspect some inadequacy in the theoretical assumption, when remarkable divergeney of the curves of $C, C_{D}$ and $\bar{C}_{A}$ is observed as in (8), (9)-1, (11) and (12)-1 of Fig. 5. However, definite merit or demerit of each model, which is still concealed, will be revealed by the analysis of the influence of medullary structure on the function.

In spite of the agreement of general tendency of $C$, there are considerable differences in the pattern of the curves between the elementary and complex models, as demonstrated in Fig. 3 and Fig. 5 . It is evident that the difference is due to the effect of structural parameters in the complex models. So far, common structural parameters have been used in all calculations. They approximately correspond to the actual structure of the inner medulla. However, the significance of the structural principle will become more obvious, if the results on models with hypothetical medullary structure are compared. The actual inner medulla is characterized by quantitative predominance of Henle's loops over the collecting tubules toward the outer border of the inner medulla. In order to elucidate its meaning we used a hypothetical set of structural parameters.

In this set, the parameters of the collecting tubule were the same as those in our previous models. The total internal surface area of Henle's loops were also left unchanged. However, exponent $h$ was made equal to $a$ or 0.39 . Consequently, the ratio $H(x) / A(x)$ becomes a constant, so that the effect of quantitative predominance of Henle's loops over the collecting tubules toward the outer border of the inner medulla is eliminated.

The parameter $H^{\prime}$ in the hypothetical set corresponding to $H$ in our standard models is determined from:

$$
H^{\prime} \int_{0}^{8}\left(e^{0.39 x}-1\right) d x=168 \int_{0}^{8}\left(e^{0.60 x}-1\right) d x
$$

Fig. 5. Some results of calculation on three complex models are demonstrated. Throughout the figures $C_{0}$ and $\nu$ are fixed to $0.020 \mathrm{~g} / \mathrm{ml}$ and $0.008 \mathrm{~g} / \mathrm{ml}$ respectively. The sets of parameters are given in tables attached to the figures. Numerals of bold style in the tables are for the parameter which loses freedom on account of fixed $C_{0}$. Structural parameters $A, a, H$ and $h$, and the flow $V$ of a Henle's loop are common to all the calculations. Total water quantity absorbed in the inner medulla is also the same and equal to $0.05 \mathrm{ml} / \mathrm{min}$ throughout the examples presented.

In (1) to (6) the combination of $\omega=0$ and $\tau=0$ is used. In (1) to (3), $K_{s}=0.2 \times 10^{-4}$, and in (4) to $(6), K_{s}=0.3 \times 10^{-4}$, the difference between the two groups being entirely due to that of $K_{s}$. Note smaller difference among $C, C_{D}$ and $\bar{C}_{A}$ in the case of larger $K_{s}$. The dotted curve in (2) corresponds to $C_{A}$ of the longest loop. This curve is drawn in order to see whether the model is consistent with the restriction that $C_{A}$ must be positive in every loop.

In (7) to (9) the combination of $\omega=-0.1$ and $\tau=0$ is used. From the pattern of the curves this condition seems compatible only with Model-1. In (9)-2 with fairly reasonable pattern, $T$ is so high that the model represents an intermediate form between (7) and (9)-1.

In (10) to (12) the combination of $\omega=-0.1$ and $r=0.1$ is used. This condition appears also appropriate only for Model-1 and for models essentially resembling Model-1. 

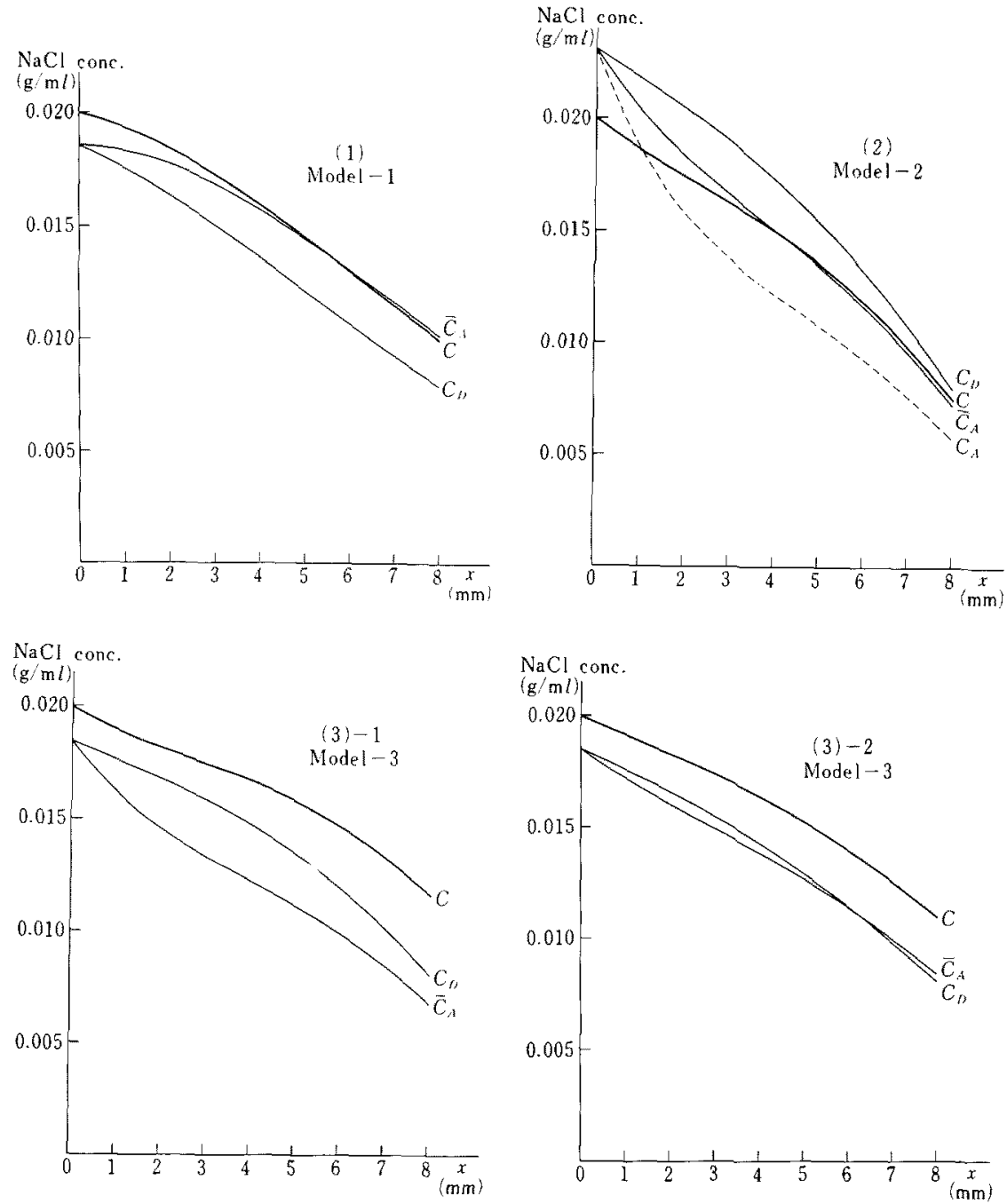

\begin{tabular}{|c|c|c|c|c|}
\hline \multirow[b]{2}{*}{ Parameter } & Model-1 & Model-2 & \multicolumn{2}{|c|}{ Model-3 } \\
\hline & (1) & (2) & (3) -1 & (3) -2 \\
\hline$A(\mathrm{~mm})$ & 114 & 114 & 114 & 114 \\
\hline$a$ & 0.39 & 0.39 & 0.39 & 0.39 \\
\hline$H(\mathrm{~mm})$ & 168 & 168 & 168 & 168 \\
\hline$h$ & 0.60 & 0.60 & 0.60 & 0.60 \\
\hline$W\left(\mathrm{~m} l / \mathrm{mm}^{2} / \mathrm{min}\right)$ & $0.9238 \times 10^{-5}$ & $0.9238 \times 10^{-5}$ & $0.9238 \times 10^{-5}$ & $0.9238 \times 10^{-5}$ \\
\hline$\omega$ & 0.0 & 0.0 & 0.0 & 0.0 \\
\hline$T(\mathrm{~g} / \mathrm{ml})$ & $2.712 \times 10^{-2}$ & $0.5 \times 10^{-2}$ & $0.5 \times 10^{-2}$ & $1.5 \times 10^{-2}$ \\
\hline$T$ & 0.0 & 0.0 & 0.0 & 0.0 \\
\hline$V(\operatorname{ml} l / \min )$ & $0.185 \times 10^{-5}$ & $0.185 \times 10^{-5}$ & $0.185 \times 10^{-5}$ & $0.185 \times 10^{-5}$ \\
\hline$K_{s}\left(\mathrm{~g} / \mathrm{mm}^{2} / \min \right)$ & $0.3 \times 10^{-4}$ & $0.2 \times 10^{-4}$ & $0.2 \times 10^{-4}$ & $0.2 \times 10^{-4}$ \\
\hline$\gamma\left(\mathrm{g} / \mathrm{mm}^{2} / \mathrm{min}\right)$ & & $0.1049 \times 10^{-6}$ & $0.1842 \times 10^{-6}$ & $0.1007 \times 10^{-6}$ \\
\hline
\end{tabular}



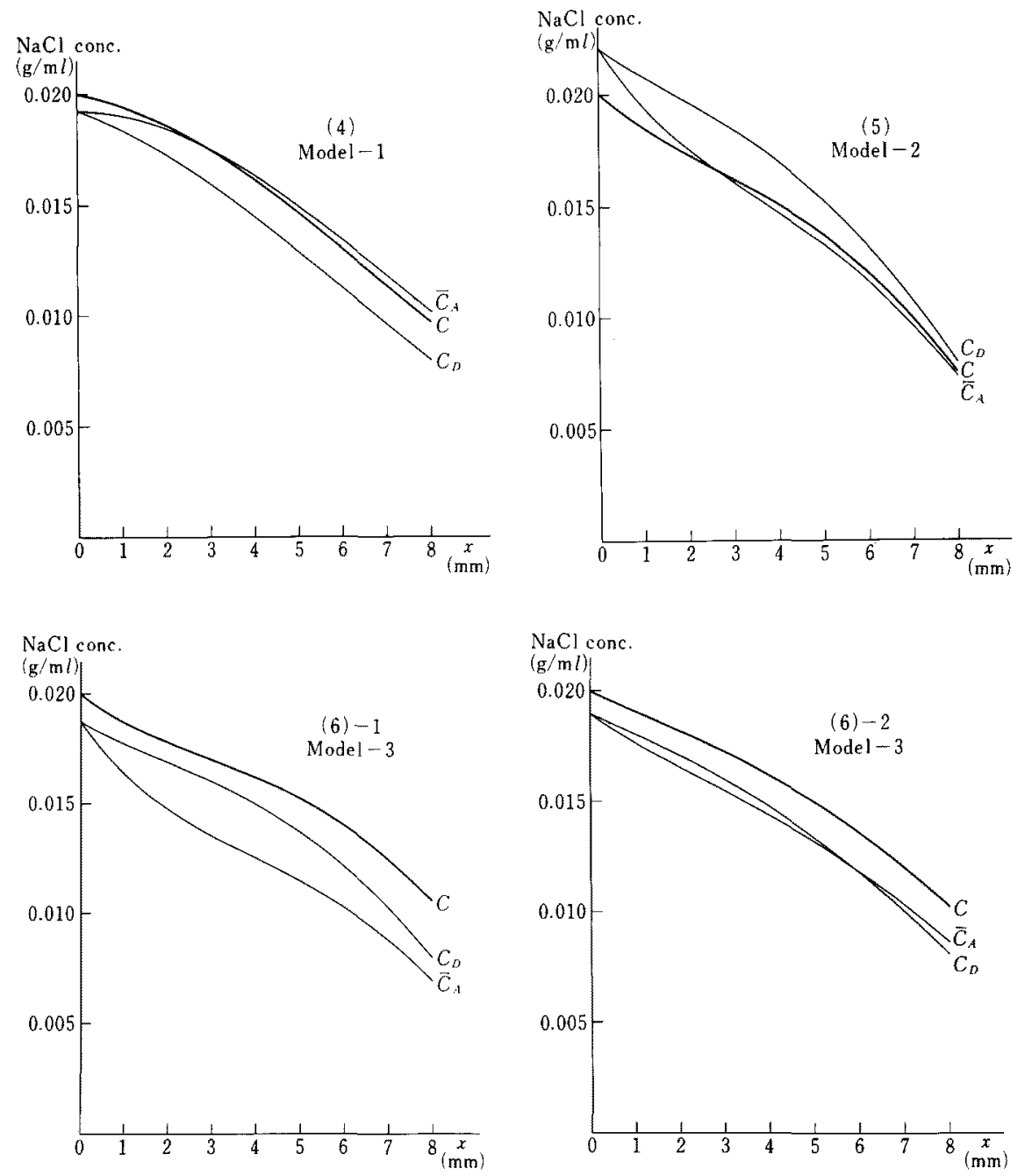

\begin{tabular}{|c|c|c|c|c|}
\hline \multirow[b]{2}{*}{ Parameter } & \multirow{2}{*}{$\begin{array}{c}\text { Model-1 } \\
(4)\end{array}$} & \multirow{2}{*}{$\frac{\text { Model-2 }}{(5)}$} & \multicolumn{2}{|c|}{ Model-3 } \\
\hline & & & (6) -1 & (6) -3 \\
\hline $\begin{array}{l}A(\mathrm{~mm}) \\
a \\
H(\mathrm{~mm}) \\
h \\
W\left(\mathrm{ml} / \mathrm{mm}^{2} / \mathrm{min}\right) \\
\omega \\
T(\mathrm{~g} / \mathrm{ml}) \\
\tau \\
V(\mathrm{ml} / \mathrm{min}) \\
K_{s}\left(\mathrm{~g} / \mathrm{mm}^{2} / \mathrm{min}\right) \\
\gamma\left(\mathrm{g} / \mathrm{mm}^{2} / \mathrm{min}\right)\end{array}$ & $\begin{array}{c}114 \\
0.39 \\
168 \\
0.60 \\
0.9238 \times 10^{-5} \\
0.0 \\
2.566 \times 10^{-2} \\
0.0 \\
0.185 \times 10^{-5} \\
0.3 \times 10^{-4} \\
-\end{array}$ & $\begin{array}{c}114 \\
0.39 \\
168 \\
0.60 \\
0.9238 \times 10^{-5} \\
0.0 \\
0.5 \quad \times 10^{-2} \\
0.0 \\
0.185 \times 10^{-5} \\
0.3 \quad \times 10^{-4} \\
\mathbf{0 . 1 0 7 4} \times \mathbf{1 0}^{-6}\end{array}$ & $\begin{array}{c}114 \\
0.39 \\
168 \\
0.60 \\
0.9238 \times 10^{-5} \\
0.0 \\
0.5 \times 10^{-2} \\
0.0 \\
0.185 \times 10^{-5} \\
0.3 \times 10^{-4} \\
\mathbf{0 . 1 9 8 9} \times 10^{-6}\end{array}$ & $\begin{array}{c}114 \\
0.39 \\
168 \\
0.60 \\
0.9238 \times 10^{-5} \\
\quad 0.0 \\
1.5 \quad \times 10^{-2} \\
0.0 \\
0.185 \times 10^{-5} \\
0.3 \times 10^{-4} \\
\mathbf{0 . 1 0 2 6} \times \mathbf{1 0}^{-6}\end{array}$ \\
\hline
\end{tabular}



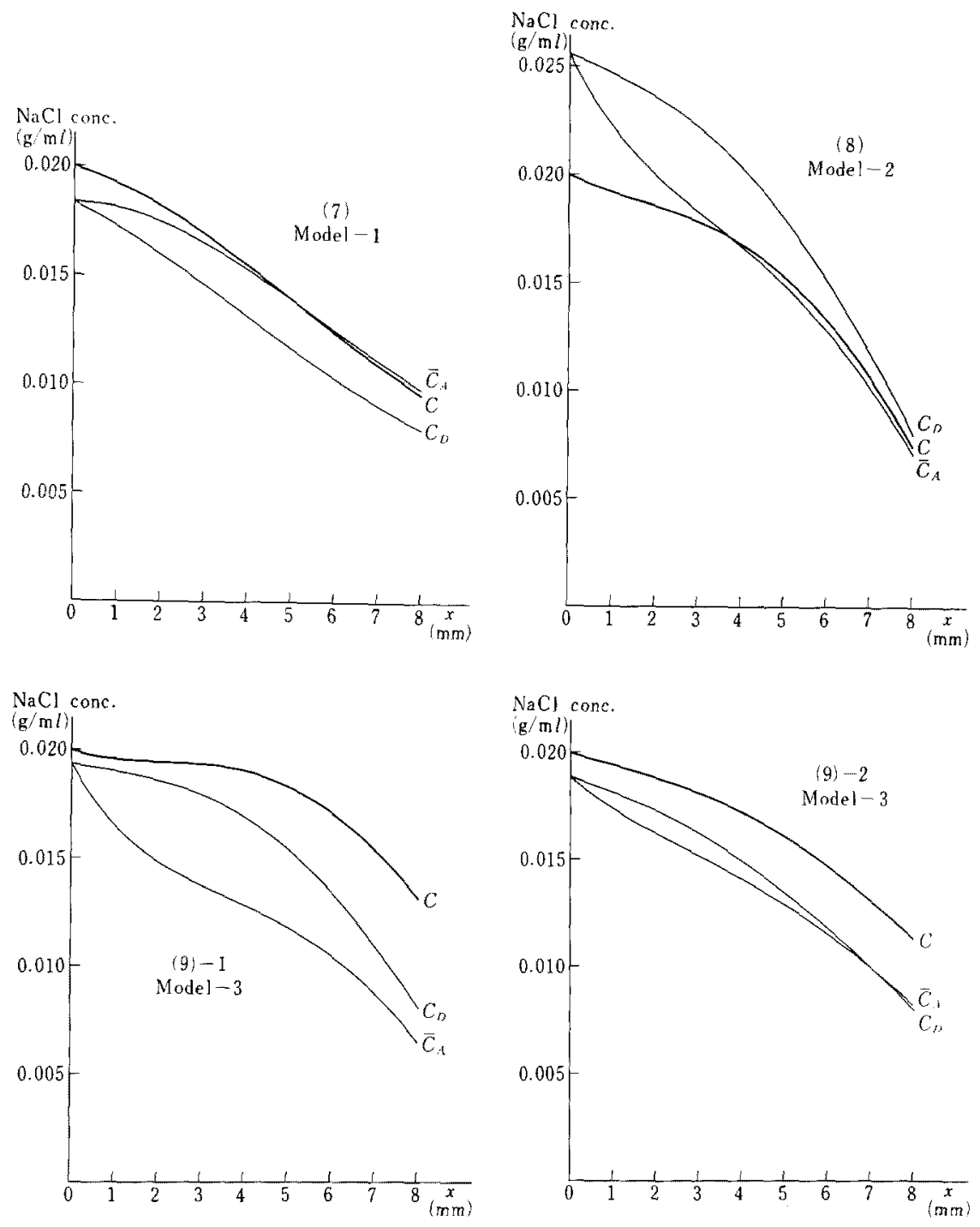

\begin{tabular}{|c|c|c|c|c|}
\hline \multirow[b]{2}{*}{ Parameter } & \multirow{2}{*}{$\begin{array}{c}\text { Model-1 } \\
(7)\end{array}$} & \multirow{2}{*}{ Model-2 } & \multicolumn{2}{|c|}{ Moeld-3 } \\
\hline & & & (9) -1 & (9) -2 \\
\hline$A(\mathrm{~mm})$ & $\begin{array}{r}114 \\
0 .\end{array}$ & $\begin{array}{l}114 \\
0.39\end{array}$ & $\begin{array}{l}114 \\
0.39\end{array}$ & $\begin{array}{l}114 \\
0.39\end{array}$ \\
\hline$H(\mathrm{~mm})$ & 168 & 168 & 168 & 168 \\
\hline$h$ & 0.60 & 0.60 & 0.60 & 0.60 \\
\hline$W\left(\mathrm{ml} / \mathrm{mm}^{2} / \mathrm{min}\right)$ & $\begin{array}{c}1.678 \times 10^{-5} \\
-0.1\end{array}$ & $1.678 \times 10^{-5}$ & $1.678 \times 10^{-5}$ & $1.78 \times 10^{-5}$ \\
\hline$T(\mathrm{~g} / \mathrm{m} l)$ & $2.443 \times 10^{-2}$ & $0.5 \times 10^{-2}$ & $0.5 \times 10^{-2}$ & $1.5 \times 10^{-2}$ \\
\hline${ }^{T} y(\mathrm{ml} / \mathrm{min})$ & $\begin{array}{c}0.0 \\
0.185 \times 10^{-5}\end{array}$ & $\begin{array}{c}0.0 \\
0.185 \times 10^{-5}\end{array}$ & $\begin{array}{c}0.0 \\
0.185 \times 10^{-5}\end{array}$ & $\begin{array}{c}0.0 \\
0.185 \times 10^{-5}\end{array}$ \\
\hline$K_{s}\left(\mathrm{~g} / \mathrm{mm}^{2} / \mathrm{min}\right)$ & $0.2 \times 10^{-4}$ & $0.2 \times 10^{-4}$ & $0.2 \times 10^{-4}$ & $0.2 \times 10^{-4}$ \\
\hline$\gamma\left(\mathrm{g} / \mathrm{mm}^{2} / \mathrm{min}\right)$ & - & $0.1401 \times 10^{-\theta}$ & $0.2474 \cdots 10^{-6}$ & $0.1200 \times 10^{-6}$ \\
\hline
\end{tabular}



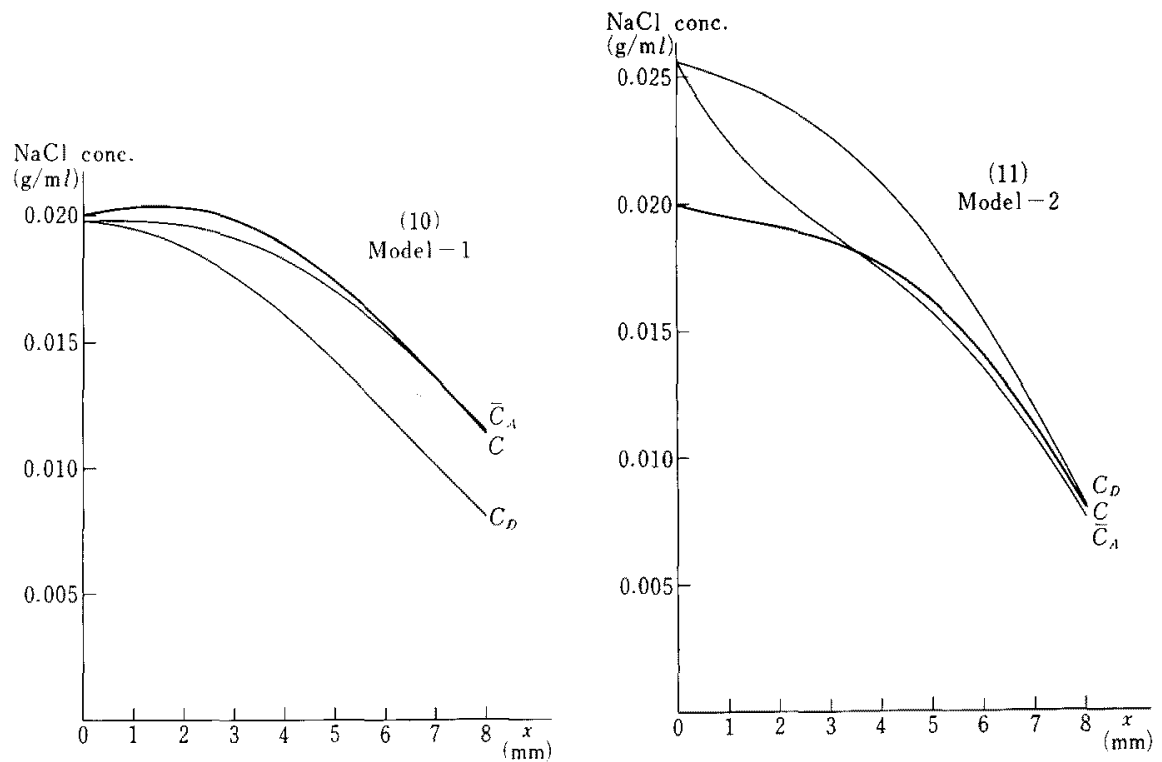

$\mathrm{NaCl}$ conc.

$(\mathrm{g} / \mathrm{ml})$

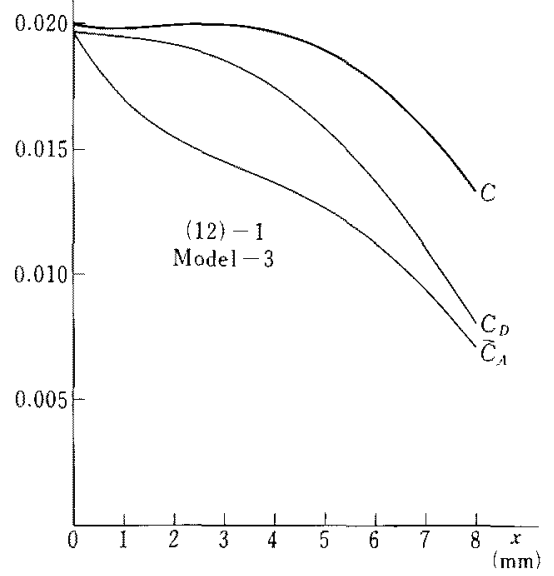

$\mathrm{NaCl}$ cone.

$(\mathrm{g} / \mathrm{ml})$

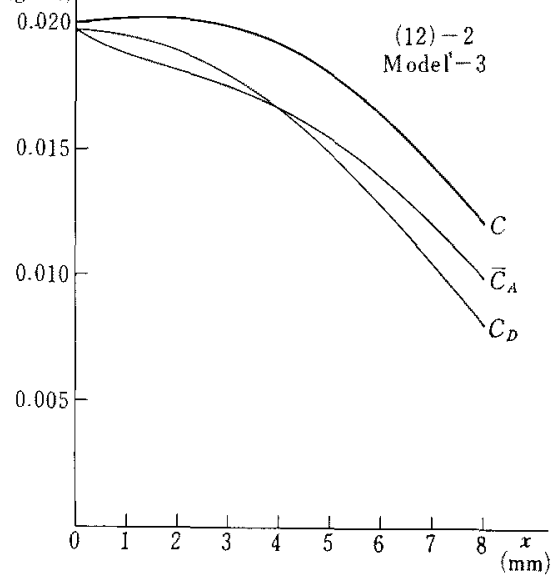

\begin{tabular}{l} 
Parameter \\
\hline$A(\mathrm{~mm})$ \\
$a$ \\
$H(\mathrm{~mm})$ \\
$h$ \\
$W\left(\mathrm{ml} / \mathrm{mm}^{2} / \mathrm{min}\right)$ \\
$\omega$ \\
$T(\mathrm{~g} / \mathrm{ml})$ \\
$\tau$ \\
$V\left(\mathrm{ml} / \mathrm{min}^{2}\right)$ \\
$K_{s}\left(\mathrm{~g} / \mathrm{mm}^{2} / \mathrm{min}\right)$ \\
$\gamma\left(\mathrm{g} / \mathrm{mm}^{2} / \mathrm{min}^{2}\right)$
\end{tabular}

\begin{tabular}{|c|c|}
\hline Model-1 & Model-2 \\
\hline$(10)$ & $(11)$ \\
\hline 114 & 114 \\
0.39 & 0.39 \\
168 & 168 \\
0.60 & 0.60 \\
$1.678 \times 10^{-5}$ & $1.678 \times 10^{-5}$ \\
-0.1 & -0.1 \\
$2.060 \times 10^{-2}$ & $0.5 \times 10^{-2}$ \\
0.1 & 0.1 \\
$0.185 \times 10^{-5}$ & $0.185 \times 10^{-5}$ \\
$0.2 \times 10^{-4}$ & $0.2 \times 10^{-4}$ \\
- & $\mathbf{0 . 1 3 3 4} \times 10^{-6}$ \\
\end{tabular}

\begin{tabular}{|c|c}
\hline \multicolumn{2}{|c}{ Model-3 } \\
\hline$(12)-1$ & $(12)-2$ \\
\hline 114 & 114 \\
0.39 & 0.39 \\
168 & 168 \\
0.60 & 0.60 \\
$1.678 \times 10^{-5}$ & $1.678 \times 10^{-5}$ \\
-0.1 & -0.1 \\
$0.5 \times 10^{-2}$ & $1.5 \times 10^{-2}$ \\
0.1 & 0.1 \\
$0.185 \times 10^{-5}$ & $0.185 \times 10^{-5}$ \\
$0.2 \times 10^{-4}$ & $0.2 \times 10^{-4}$ \\
$\mathbf{0 . 2 3 5 6} \times 10^{-6}$ & $\mathbf{0 . 8 4 5 4} \times 10^{-6}$
\end{tabular}




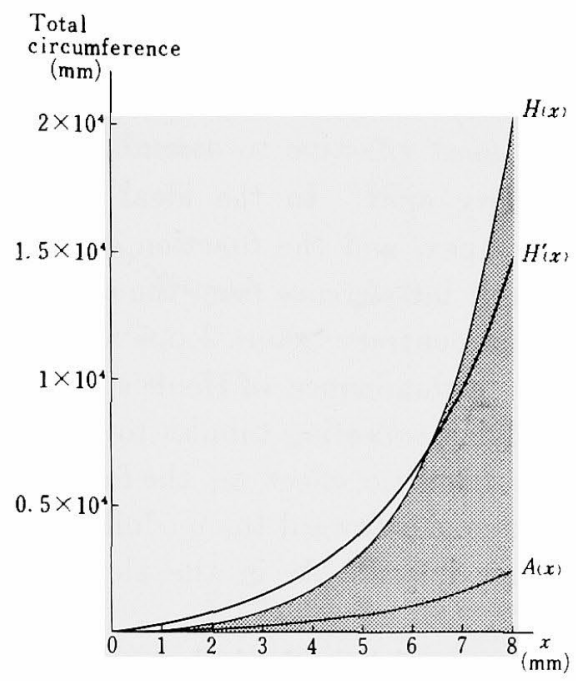

Fig. 6. The meaning of the hypothetical structural parameters used in the present calculation is diagrammatically explained. $H(x)$ with shadowed area under the curve shows the actual pile of Henle's loops. Thick curve $H^{\prime}(x)$ corresponds to the hypothetical pile of the loops. The areas under $H(x)$ and $H^{\prime}(x)$ are the same, or the total internal surface areas of the loops are equal in both piles. Since $H^{\prime}(x)$ is lower than $H(x)$ at $x=8 \mathrm{~mm}$, the number of loops is smaller and the average length of loops is longer in the pile of $H^{\prime}(x) . \quad A(x)$ represents the total internal circumference of the collecting tubules. The ratio $H^{\prime}(x) / A(x)$ is a constant independent of $x$, because the exponents are made the same in both functions. The quantitative proportion of Henle's loops and collecting tubules is thus uniform over the whole range of $x$.

and the equation for Henle's loops in the hypothetical model becomes accordingly:

$$
H^{\prime}(x)=682\left(e^{0 ; 39 x}-1\right) .
$$

The configuration of Henle's loops corresponding to the hypothetical parameter set is illustrated in Fig. 6. The total internal surface area of the loops is unchanged, but its distribution is relatively shifted toward the medullary apex.

The results of computation are presented in Fig. 7, the other parameters being the same as those in (1), (2) and (3)-1 of Fig. 5. It is especially worthy of notice that in the case of the hypothetical parameter set $C_{0}$ is evidently lowered in Model-1, but distinctly elevated in Model-2 and Model-3. The result is important because it demonstrates very clearly what the influence of the medullary structure is. In Model-1, in which active sodium transport is not assumed on Henle's loops, the structural principle of the actual inner medulla acts synergetically in raising interstitial sodium concentration toward the medullary apex. In this model, Henle's loops are a scdium-depriving system, so that the quantitative predominance of the loops over the collecting tubule toward the outer medulla reinforces the effect of individual loops in producing the observed gradient of sodium concentration. The result seems to support the theoretical assumption in Model-1 as the most appropriate one. 
In Model-2 and Model-3, the effect of individual loops is depressed in the actual pile of Henle's loops. In these models Henle's loops are a sodium-supplying system and sustain high sodium concentration of the interstitial tissue. Under this condition, it would be most effective to assemble as many Henle's loops as possible toward the medullary apex. In the ideal configuration, all the loops would attain the medullary apex, and the function of individual loops would be integrated without unfavorable interference from the structure of the pile. In the actual inner medulla, on the contrary, short loops are overwhelmingly large in number. The quantitative predominance of Henle's loops as a sodium-supplying system over the water-supplying collecting tubules toward the outer border of the inner medulla exerts an antagonistic effect on the formation of gradient of interstitial sodium concentration rising toward the medullary apex. The result makes Model-2 and Model-3 rather improbable in the simulation of medullary function.

In our models with the set of hypothetical structural parameters, the total internal surface area of Henle's loops is the same as that of the standard models. On account of this limitation, the number of the loops is reduced. This is immediately conceived from Fig. 6. Consequently, the total flow in the pile of Henle's loops is reduced, if the flow of each loop is not changed. To make the total flow unchanged, the number of loops must be the same even in the case of hypothetical structural parameters. The total internal surface area of Henle's loops is inevitably enlarged, leading to increased contact with the interstitial tissue. It is easily comprehended that such a parameter set only exaggerates the effects already demonstrated in Fig. 7 , and we do not think it necessary to present the result of calculations.

\section{DIsctssion}

The analysis of the influence of medullary structure on the medullary function revealed that Model-1 was adequate in the simulation of the function of the inner medulla. In the elementary model with a single Henle's loop a variety of theoretical assumptions appeared to be compatible with experimental observations. However, some of them were conflicting with the structural principle of the medulla and could not be maintained in the simulation of the total medullary function.

The major characteristic of the structure of the inner medulla is the quantitative predominance of Henle's loops over the collecting tubules toward the outer

Fig. 7. The curves obtained with a hypothetical set of structural parameters are demonstrated. The other parameters including $T$ and $\gamma$ are the same as those in (1) to (3)-1 of Fig. 5 corresponding to each model. See attached table for the value of parameters. The initial condition $y$ is also the same. The dotted curves represent $C$ in (1) to (3)-1 of Fig. 5 according to each model. They are drawn for comparison and for easy understanding of the effect of hypothetical structural parameters. Note depressed $C$ in Model-1 and raised $C$ in Model-2 and Model-3. 
Functional Model of Renal Medulla
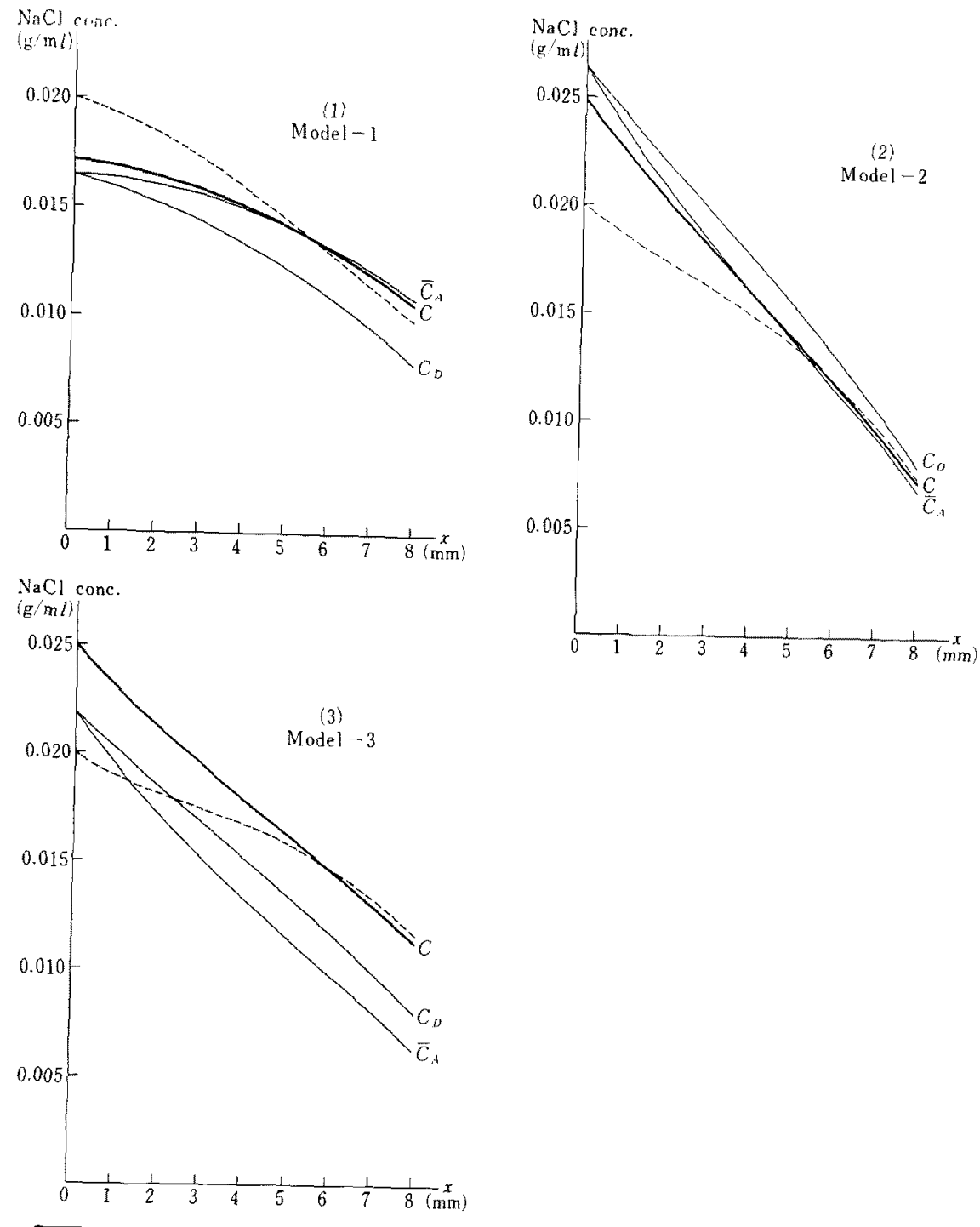

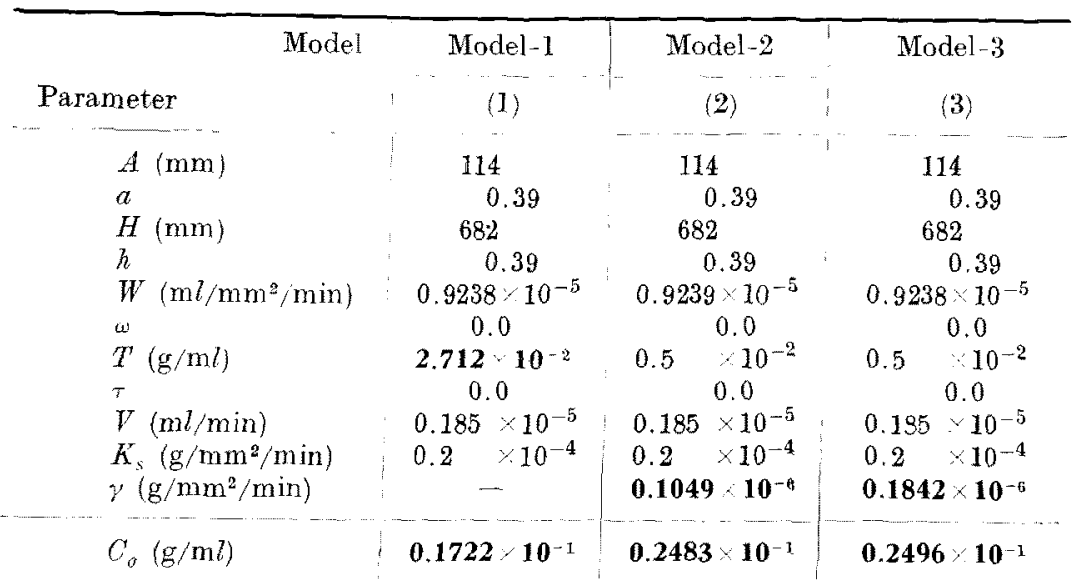


border. This principle is effective in producing the pattern of interstitial sodium concentration rising toward the medullary apex, when Henle's loops are a sodium-depriving system. On the contrary, the medullary structure is contradictory to the assumption of Henle's loops as a sodium-supplying system. We think that the theoretical assumptions, whatever they may be, must first of all satisfy the condition that Henle's loops are a sodium-depriving system.

This requirement does not mean immediately that every active sodium transport should be denied to Henle's loops. It is the final balance that is important, and it is determined by the algebraic sum of active transport and passive exchange. Weak active sodim transport, even if pressnt, may be counteracted by passive sodium transfer to the opposite direction. In any way, active sodium transport cannot be so strong as to sustain and determine sodium concentration of the interstitial tissue without help of much larger sodium supply from the collecting tubules, For this reason, we camnot accept the counter-current hypothesis of Henle's loops as a plausible explanation of medull iry function.

In the model with Henle's loops as a sodium-depriving system, a large quantity of sodium must be afforded from the collecting tubules to counterbalance the effect of water absorption and to sustain high intsrstitial sodium concentration. The source of sodium is again to be sought in glomerular filtrate flowing into the collecting tubules of the imer medull. On account of this requirement, sodium concentration at the proximal end of the collecting tubules in the imner medulla becomes distinctly hypertonic in our model. It exceeds at least $0.01 \mathrm{~g} / \mathrm{ml}$ with our set of parameters and under the condition that $0.05 \mathrm{ml} / \mathrm{min}$ of water is absoibed in the inner medulla and final urinary volume is not much larger than $0.05 \mathrm{ml} / \mathrm{min}$ per kidney. The assumption of hypertonic sodium concentration at the proximal collecting tubule is supported by the experimental study of Hilger et al. However, an exact determination of the concentration still awaits further investigations.

If sufficient hypertonicity of sodium as required in the present study is not demonstrated, two possibilities may be taken into consideration. One of them is that Henle's loops have an ability of weak active sodium transport, which is by itself not enough to sustain high interstitial sodium concentration, but has an effect of sparing the sodium quantity to be afforded by the collecting tubules. In this case, Henle's loops play a subsidiary part in the formation of sodium concentration gradient of the interstitial tissue. Even in this case Henle's loops would not necessarily constitute a counter-current multiplier system. It would be at least equally probable that the ascending limbs of Henle's loops alone have active sodium transport.

The other possibility is that water absorption from the collecting tubules of the imner medulla is estimated too high in our model in comparison with presumed final urinary volume. If smaller quantity of water is absorbed in the inner medulla, sodium quantity necessary to sustain high interstitial sodium concentration is lowered and the requirement of sodium hypertonicity at the proximal collecting tubules is moderated. The latter effect is also expected, if final urinary volume is 
larger than that presumed in our model.

In the present study, the outer medulla could not be submitted to theoretical analysis. This was mainly due to much greater complexity of the structure of the outer medulla. Beside the tissue elements of the inner medulla, distal straight portion of the proximal tubules and thick segments of Henle's ascending limbs constitute the outer medulla. Moreover, we had not enough information about the role of each constituent. The number of possible combinations of roughly permissible theoretical assumptions would be too large, and structural analysis could not contribute much to elucidating the mechanism of the medullary function.

However, our conclusion on the function of the inner medulla imposes some restriction to the possible role of the outer medulla. It is generally accepted that glomerular filtrate in the distal convolution of the renal cortex is rather hypotonic. On the other hand, hypertonic sodium concentration is required from our theoretical analysis in the collecting tubule at the border of inner and outer medullas. Consequently, sodium concentration must be elevated in the course of urinary passage through the outer medulla. This will be achieved by water transport from the collecting tubule leaving sodium behind. In spite of this predominant water supply, the sodium concentration of the interstitial tissue of the outer medulla is to be sustained at a relatively high level. The necessary sodium supply is most reasonably sought in the thick segment of Henle's ascending limb, which is probably endowed with a strong ability of active sodium transport. Rich cytoplasm of this portion makes an assumption of biological work requiring large energy consumption reasonable. Large sodium quantity carried by Henle's ascending limbs from the inner medulla is also compatible with the assumption that Henle's thick segments are the source of sodium in sustaining high sodium concentration of the interstitial tissue in the outer medulla.

In this hypothesis, we think that there is an intramedullary circulation of

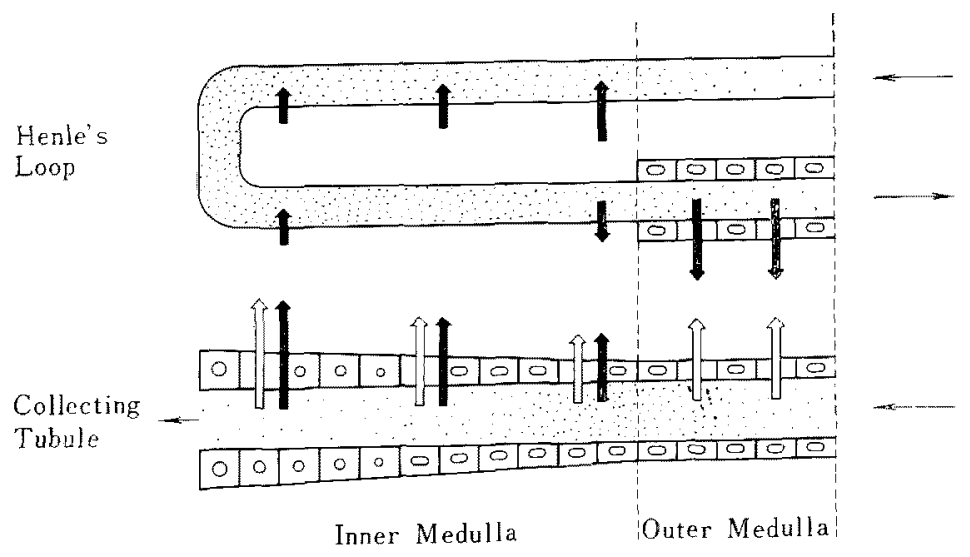

Fig. 8. Intramedullary sodium circulation proposed for the mechanism of urinry concentration in the medulla is illustrated. I and if indicate water and sodium transfer respectively. The density of dots in Henle's loop and collecting tubule roughly corresponds to presumed sodium concentration. 


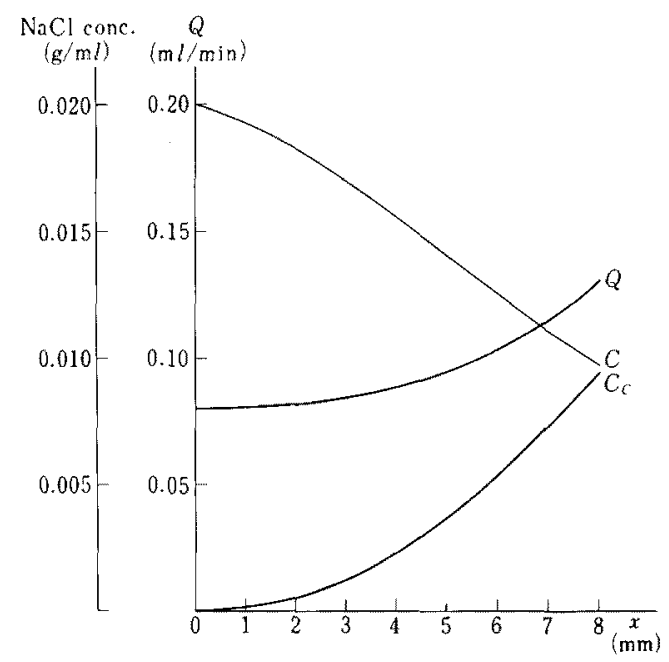

Fig. 9. Total urinary flow $Q$ and $\mathrm{NaCl}$ concentration $C_{c}$ of the collecting tubules of the inner medulla are demonstrated with Model-1 in Fig. 5-(7). The combination of $\omega=-$ 0.1 and $r=0$ is used. The final urinary volume is $0.08 \mathrm{ml} / \mathrm{min}$ and NaCl concentration in the final urine is assumed to be zero in the calculation of this figure.

sodium as illustrated in Fig. 8. The outer and inner medullas act cooperatively in producing the sodium circulation. Apparently water absorption from the collecting tubule is a process secondary to the sodium transfer and more or less passive in its nature. It is not known, however, if water absorption is strictly proportional to the difference of sodium concentrations in and outside the collecting tubule. Such a condition is not dealt with in the present analysis. Whether a theoretical model is possible under this condition awaits still further studies.

According to the present hypothesis, the function of the collecting tubule in regard to active sodium transport must be different between the inner and outer medullas. In the latter, active sodium transport is expected to be absent or minimal. Because histological appearance of the collecting tubules changes continuously from the inner to outer medulla, an abrupt change in their function seems rather improbable. It would be justified to assume that the active sodium transport of the collecting tubule of the inner medulla gradually decreases toward the outer medulla. In this respect, the combination of $\omega=-0.1$ and $\tau=0$ seems to be a fairly good approximation.

In Fig. 9, an example of calculation is presented demonstrating possible patterns of total urinary flow and $\mathrm{NaCl}$ concentration in the collecting tubules of the inner medulla. The $\mathrm{NaCl}$ concentration of the interstitial tissue is still higher than that of the collecting tubule at the proximal inner medulla. The assumption of passive water transfer by the difference of $\mathrm{NaCl}$ concentrations in and outside the tubule is sustained, if water transfer proportional to the difference is not postulated. 


\section{Acknowledgment}

The computation in the present study was performed with NEAC-2230 of the Computing Center, Tohoku University. We are indebted to Miss I. Ito for her technical assistance.

\section{References}

1) Hargitay, B. \& Kuhn, W. Das Multiplikationsprinzip als Grundlage der Harnkonzentrierung in der Niere. Z. Elektrochem., 1951, 55, 539-558.

2) Kuhn, W. \& Ramel, A. Aktiver Salztransport als möglicher (und wahrseheinlicher) Einzeleffekt bei der Harnkonzentrierung in der Niere. Hele. chim. Acta, 1959, 42, 628-660.

3) Wirz, H. Der osmotische Druck des Blutes in der Nierenpapille. Helv. physiol. Acta, $1953,11,20-29$.

4) Marsh, D. J. \& Solomon, S. Analysis of electrolyte movement in thin Henle's loops of hamster papilla. Amer. J. Physiol., 1965, 208, 1119-1128.

5) Hilger, H. H., Klümper, J. D. \& Ullrich, K.J. Wasserrückresorption und Ionentransport durch die Sammelrohrzellen der Säugetierniere. Pflugers Arch. ges. Physiol., 1958, 267, 218-237.

6) Sasaki, Y., Takahashi, T. \& Suwa, N. Quantitative structural analysis of the inner medulla of rabbit kidney. Tohoku $J$ exp. Med., 1968, 98, 21-32. 